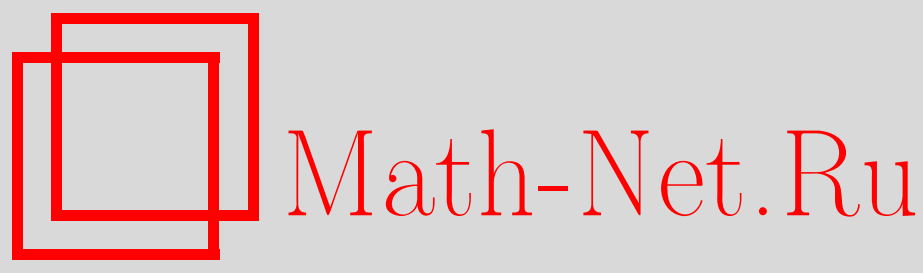

А. Б. Калмынин, О конференции памяти А. А. Карацубы по теории чисел и приложениям, Совр. пробл. матем., 2017, выпуск 24, 7-45

DOI: https://doi.org/10.4213/spm67

Использование Общероссийского математического портала Math-Net.Ru подразумевает, что вы прочитали и согласны с пользовательским соглашением http://www . mathnet.ru/rus/agreement

Параметры загрузки:

IP: 54.162 .27 .143

26 апреля 2023 г., 14:48:30

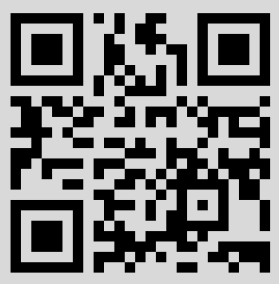




\title{
О конференции памяти А. А. Карацубы по теории чисел и приложениям
}

\author{
А. Б. Калмынин \\ Национальный исследовательский университет "Высшая школа экономики", г. Москва \\ alkalb1995cd@mail.ru
}

\section{1. Введение}

Цель «Конференции памяти А.А. Карацубы по теории чисел и приложениям» - представление новых и важных результатов в разных направлениях теории чисел таких, как: аналитическая теория чисел, диофантовы приближения, геометрия чисел, а также по приложениям теории чисел в других разделах математики. Участниками стали математики из России, стран СНГ и Европы. Первые две конференции прошли в январе 2014 г. и 2015 г. и были однодневной и двухдневной соответственно. Одной из важных задач конференции является общение между участниками в целях обмена идеями и создания возможности для сотрудничества, в частности, в тех областях, которые связаны с исследованиями А. А. Карацубы.

Третья конференция памяти Анатолия Алексеевича Карацубы по теории чисел и приложениям проходила с 28 по 30 января 2016 г. в Математическом институте им. В. А. Стеклова Российской академии наук (28 и 29 января) и на механико-математическом факультете Московского государственного университета имени М. В. Ломоносова (30 января) в Москве.

В Организационный комитет вошли Е. А. Карацуба (ВЦ РАН), М. А. Королёв (МИАН), И. С. Резвякова (МИАН) и В.Н. Чубариков (МГУ). В течение трех дней на конференции было сделано 30 докладов. ${ }^{1}$

\section{2. Обзор докладов 2016 года}

Й. Ф. Андерссон (Малардален - государственный университет, Вестерос), Универсальность дзета-функиий Эпштейна "по решеткам"

В 1975 г. С. М. Воронин доказал свою знаменитую теорему об универсальности дзета-функции.

Теорема 1 (Универсальность дзета-функции Римана). Пусть $K=\{s \in \mathbb{C}:|s-3 / 4| \leqslant r\}$ для некоторого $r<1 / 4$. Пусть $f$ - не обращающаяся в нуль голоморфная на внутренности $K$ $u$ продолжающаяся непрерывно на границу $K$ функция. Тогда для всякого положительного $\varepsilon$ имеет место неравенство

$$
\liminf _{T \rightarrow+\infty} \frac{1}{T} \mu\left\{t \in[0 ; T]: \max _{s \in K}|\zeta(s+i t)-f(s)|<\varepsilon\right\}>0 .
$$

Позднее оказалось, что в качестве $K$ в этой теореме можно выбирать любой компакт со связным дополнением в правой половине критической полосы.

Работа выполнена при поддержке Лаборатории зеркальной симметрии НИУ ВШЭ, гранта Правительства PФ (договор № 14.641.31.0001), фонда поддержки молодых ученых «Конкурс Мёбиуса», a также фонда Simons Foundation.

${ }^{1} \mathrm{C}$ аннотациями и видеозаписями докладов можно ознакомиться на официальной странице конференции http://www.mathnet.ru/conf773. 
Позднее были получены результаты об универсальности в различных семействах $L$-функций, например, следующий.

Теорема 2 (Б. Багчи, 1981, Универсальность по $L$-функциям Дирихле). $B$ условиях предъдущей теоремы, для произвольно малого $\varepsilon>0$ выполнено

$$
\liminf _{p \rightarrow+\infty} \frac{1}{p-1} \#\left\{\chi \bmod p: \max _{s \in K}|L(s, \chi)-f(s)|<\varepsilon\right\}>0,
$$

где р пробегает простые числа.

Доказана также универсальность по квадратичным характерам.

Теорема 3 (Х. Мишоу, Х. Нагоши, 2006). Для простого числа р положим

$$
\chi_{p}(\cdot)=\left(\frac{\cdot}{p}\right) .
$$

Если $\Omega$ - односвязная симметричная относительно вещественной оси область в полосе $\{s$ : $1 / 2<\operatorname{Re} s<1\}$, то для всякого $\varepsilon>0$, любого компакта $K \subset \Omega$ и любой не имеющей нулей голоморфной $f$ на $\Omega$ с вещественными положительными значениями на $\Omega \cap \mathbb{R}$ справедливо неравенство

$$
\liminf _{N \rightarrow+\infty} \frac{1}{\pi(N)} \#\left\{p \leqslant N: \max _{s \in K}\left|L\left(s, \chi_{p}\right)-f(s)\right|<\varepsilon\right\}>0 .
$$

Докладчиком в соавторстве с А. Сёдергреном были доказаны результаты об универсальности "по решеткам". Дзета-функция Эпштейна $E(L, s)$, отвечающая решетке $L \subset \mathbb{R}^{n}$, задается рядом

$$
\sum_{m \in L \backslash\{0\}}\|m\|^{-2 s},
$$

где $\|\cdot\|$ - норма на $L$. Хорошо известно, что $E(L, s)$ удовлетворяет функциональному уравнению

$$
F_{n}(L, s)=F_{n}\left(L^{*}, \frac{n}{2}-s\right)
$$

в котором

$$
F_{n}(L, s)=\pi^{-s} \Gamma(s) E(L, s),
$$

а $L^{*}$ - двойственная решетка. Справедливы следующие теоремы.

Теорема 4 (Андерссон, Сёдергрен, 2015). Пусть $\Omega$ - односвязная область в правой половине критической полосы, симметричная относительно $\mathbb{R}$. Для всякого положительного $\varepsilon$, компакта $K \subset \Omega$ и голоморфной функиии $f$ на $\Omega$, вещественной на $\Omega \cap \mathbb{R}$, выполняется неравенство

$$
\liminf _{n \rightarrow+\infty} \mu_{n}\left\{L \in X_{n}: \max _{s \in K}\left|2^{s-1} V_{n}^{-s} E\left(L, \frac{n s}{2}\right)-f(s)\right|<\varepsilon\right\}>0,
$$

где $X_{n}$ - множество всех $n$-мерных решеток кообъема 1 и $\mu_{n}$ - мера Зигеля на $X_{n}$, нормированная так, чтобы стать вероятностной.

Теорема 5 (Андерссон, Сёдергрен, 2015). Пусть $\Omega$ - односвязная область в полуплоскости $\operatorname{Re} s>1 / 2$, симметричная относительно $\mathbb{R}$. Если $f$ - голоморфная на $\Omega$ функиия, вещественнозначная на $\Omega \cap \mathbb{R}, K$ - компакт в $\Omega$ и $\varepsilon>0$, то имеет место неравенство

$\liminf _{n \rightarrow+\infty}\left(\mu_{n} \times \mu_{n}\right)\left\{\left(L_{1}, L_{2}\right) \in X_{n}^{2}: \max _{s \in K}\left|2^{s-1} V_{n}^{-s} E\left(L_{1}, \frac{n s}{2}\right)-2^{s-1} V_{n}^{-s} E\left(L_{2}, \frac{n s}{2}\right)-f(s)\right|<\varepsilon\right\}>0$. 
Последняя теорема интересна тем, что это первый пример универсальности в полуплоскости, а не в полосе. Доказательство основано на стандартных утверждениях об аппроксимациях многочленами Дирихле и результатах из диссертации Сёдергрена, содержащей вероятностные утверждения о нормах элементов решеток.

А. И. Аптекарев (Институт прикладной математики им. М. В. Келдыша РАН, г. Москва), О функииональном аналоге теоремы Туэ-Зигеля-Рота

Зададимся вопросом, насколько точно можно приблизить данное алгебраическое число $\alpha$ рациональным числом с ограниченным знаменателем. Хорошо известны как верхние, так и нижние оценки для точности такого приближения: например, по теореме Гурвица, для всякого иррационального $\alpha$ существует бесконечно много целых $p$ и $q$ таких, что

$$
\left|\alpha-\frac{p}{q}\right|<\frac{1}{\sqrt{5}} q^{-2} .
$$

С другой стороны, по теореме Лиувилля, если $\alpha$ - алгебраическое число $k$-й степени с $k \geqslant 2$, то существует такая константа $C(\alpha)$, что для всех целых $p$ и $q$ имеет место неравенство

$$
\left|\alpha-\frac{p}{q}\right| \geqslant C(\alpha) q^{-k} .
$$

Последнее утверждение может быть усилено.

Теорема 1 (А. Туэ, 1909). Если $\alpha$-алгебраическое число степени $k$, то для всякого $\varepsilon>0$ и всех р и я выполнено

$$
\left|\alpha-\frac{p}{q}\right| \gg_{\varepsilon} q^{-k / 2-1-\varepsilon} .
$$

Теорема 2 (К.Л. Зигель, 1921, Ф.Д. Дайсон, А. О. Гельфонд, 1947-1948). В условиях предыдущей теоремы для всех целых $p, q$ выполнена оценка

$$
\left|\alpha-\frac{p}{q}\right| \gg_{\alpha} q^{-\sqrt{2 k}} .
$$

Наконец, верно следующее.

Теорема 3 (К.Ф. Рот, 1955). Для всякого алгебраического а и произвольного фиксированного $\varepsilon>0$ существует константа $C$ такая, что

$$
\left|\alpha-\frac{p}{q}\right| \geqslant \frac{C}{q^{2+\varepsilon}}
$$

Несмотря на то, что последняя теорема почти неулучшаема, некоторые вопросы о приближениях алгебраических чисел остаются открытыми. Например, не известно, ограничены ли неполные частные их цепных дробей. Так, вычисления, проведенные независимо А. Д. Брюно (1964), С. Ленгом (1972) и докладчиком (1975), показывают, что среди неполных частных $a_{n}$ числа $2^{1 / 3}$ встречаются числа $14=a_{10}, 543=a_{36}, 7451=a_{572}$ и т.д. (хотя их неограниченность не доказана).

При приближении же алгебраических функций рациональными наблюдается некоторый совершенно новый эффект, а именно, выполнен аналог сильной формы теоремы 3.

Пусть

$$
f=\sum_{n=0}^{+\infty} f_{k} z^{-(k+1)}
$$


Для такого ряда $f$ введем величины

$$
\nu_{n}(f)=\sup \left\{\operatorname{ord}_{\infty}(f-r): r \in R_{n}\right\},
$$

где $R_{n}$ - рациональные функции степени не выше $n, \operatorname{ard} \operatorname{ord}_{\infty}(g)$ обозначает степень первого ненулевого слагаемого при разложении $g$ по степеням $z^{-1}$.

Гипотеза Э. Р. Колчина (1959) утверждает, что для всякого $f$, удовлетворяющего алгебраическому дифференциальному уравнению, выполнено равенство

$$
\lim _{n \rightarrow \infty} \frac{\nu_{n}(f)}{n}=2 .
$$

Гипотеза Колчина была доказана С. Учиямой и Г. Шталем. Позднее А. А. Гончаром (1978) и Д. В. и Г. В. Чудновскими (1984) была высказана более сильная гипотеза.

ГипотезА 1. Для всякой алгебраической функции $f$ существует такое $C(f)$, что

$$
\nu_{n}(f) \leqslant 2 n+C(f) .
$$

Это утверждение в некоторых естественных ограничениях на функцию $f$ было доказано в совместной работе докладчика и М. Ятцелева. Более того, справедлива

Теорема 4. Гипотеза 1 верна для всех функций, удовлетворяющих алгебраическому дифференциальному уравнению. Кроме того, требуется, чтобы точки ветвления были в общем положении.

Р. Н. Бояринов (Московский государственный университет имени М. В. Ломоносова, механико-математический факультет), Метод моментов и его приложения в теории чисел

Пусть $\xi$ - случайная величина на вероятностном пространстве $(\Omega, \Sigma, \mathbb{P})$. Рассмотрим последовательность ее моментов

$$
m_{n}(\xi)=\int_{\Omega} \xi^{n} d \mathbb{P}
$$

При некоторых условиях на случайную величину последовательность моментов полностью определяет ее распределение. Первые результаты в этом направлении были получены П. Л. Чебышёвым и А. А. Марковым.

В теории чисел часто необходимо выводить эффективные оценки на сходимость случайных величин из оценок на сходимость четных моментов. В связи с этим возникают следующие две задачи:

1) оценить расстояние между функциями распределения двух случайных величин, если известны расстояния между их четными моментами;

2) оценить расстояние между их дробными моментами при тех же условиях.

В 1983 г. эти задачи были решены А. Гошем в случае аргумента дзета-функции Римана для доказательства предельной теоремы Сельберга.

В 2006 г. аналогичные результаты были получены А. А. Карацубой и М. А. Королёвым для коротких интервалов критической прямой.

Помимо нормального распределения в задачах теории чисел также очень часто возникает и экспоненциальное распределение. В связи с этим может быть полезна следующая теорема, доказанная докладчиком.

ТеОрема 1. Пусть $\xi_{1}, \xi_{2}, \ldots, \xi_{n}, \ldots$ - последовательность случайных величин. Пусть для всех достаточно больших $n$ существует $N(n)$ такое, что при $1 \leqslant k \leqslant N(n)$ выполнено равенство

$$
m_{2 k}\left(\xi_{n}\right)=\sigma_{2 k}\left(1+\frac{\theta}{f(n)}\right)
$$


где $f(n) \rightarrow+\infty u|\theta| \leqslant 1$. Тогда справедливо равенство

$$
F_{\xi_{n}}(a)=F(a)+R_{n}
$$

$u$

$$
\left|R_{n}\right| \leqslant 6\left(\frac{134(\ln N(n)+1)}{\sqrt{N(n)}}+2^{-N(n)}+\frac{3^{N(n)}}{f(n)}\right) .
$$

Здесь $F(a)=1-e^{-a^{2}}$, если $\sigma_{2 k}=k !, u F(a)$ - функиия распределения нормального распределения, если $\sigma_{2 k}=(2 k) ! /\left(2^{k} k !\right)$.

Аналогичное утверждение верно и в более общем случае.

ТеОрема 2. Пусть выполнены условия предыдущей теоремы с $0<\sigma_{2 k} \leqslant(C k)^{k(2-\delta)} \partial л я$ некоторых $\delta, C>0$. Пусть $\sigma_{2 k}$ - моменты некоторой случайной величины с функцией распределения $F(x)$, удовлетворяющей условию Липшища с константой L. Тогда для достаточно больших $n$ имеет место неравенство

$$
\left|F_{\xi_{n}}(a)-F(a)\right| \leqslant \max \left(2 B^{2}, 6 L\right)\left(\frac{224 C(\ln N(n)+1)}{N^{\delta / 2}}+3^{-N(n)}+\frac{N^{2 B N}}{f(n)}\right)
$$

где $B=[4 C]+1$.

Похожие результаты получаются и для дробных моментов.

ТЕОРема 3. Пусть для всех достаточно больиих $n$ и всех иельх $1 \leqslant k \leqslant[\rho \ln f(n)]+1$, где $0.1 \geqslant \rho>0$, выполнено

$$
m_{2 k}\left(\xi_{n}\right)=\sigma_{2 k}\left(1+\frac{\theta}{f(n)}\right)
$$

$c|\theta| \leqslant 1$.

Тогда для всех достаточно больших $n$ и всех $a \in(0,0.5 \rho \ln f(n)]$ выполнено равенство

$$
m_{a}\left(\xi_{n}\right)=\mu(a)+\theta R_{n},
$$

где

$$
\mu(a)= \begin{cases}2^{0.5 a} \Gamma(0.5 a+0.5) \pi^{-0.5}, & \text { если } \sigma_{2 k}=\frac{(2 k) !}{2^{k} k !}, \\ \Gamma(0.5 a+1), & \text { если } \sigma_{2 k}=k !,\end{cases}
$$

и остаточный член имеет вид

$$
R_{n}= \begin{cases}\frac{2^{11-\delta}}{a}\left(\frac{2^{22} \ln \ln f(n)}{\rho \ln f(n)}\right)^{\frac{a+1+\delta}{2}} & n p u \quad 0<a<30, \\ 2^{7} \mu(a)\left(\frac{2^{12+2 \delta} a^{2} \ln (\sqrt{\ln f(n)} / a)}{\rho \ln f(n)}\right)^{\frac{a+1+\delta}{2}} & n p u \quad 30 \leqslant a \leqslant \frac{\rho}{2^{16+2 \delta}} \sqrt{\ln f(n)}, \\ 2^{2+\delta} \mu(a) e^{-\frac{\rho \sqrt{\ln f(n)}}{2^{20+2 \delta}}} & n p u \quad \frac{\rho}{2^{16+2 \delta}} \sqrt{\ln f(n)}<a \leqslant 0.5 \rho \ln f(n) .\end{cases}
$$

Из теорем 1-3 можно выводить разные утверждения о лакунарных тригонометрических суммах, например, следующее. 
ТеОрема 4. Пусть $\omega \in[0,1], n$ - натуральное число и $u_{k}$ - последовательность натуральных чисел с $u_{k+1} / u_{k} \geqslant \beta>1$. Положим $S(\omega ; n)=\sum_{k \leqslant n} e^{2 \pi i \omega u_{k}}$. Тогда для всех достаточно больших $n$ и любого $\lambda>0$ функция распределения случайной величинь $|S(\omega ; n) / \sqrt{n}|$ удовлетворяет соотношению

$$
F_{n}(\lambda)=1-e^{-\lambda^{2}}+\frac{4600 \theta \sqrt{\ln (2 \beta /(\beta-1))} \ln \ln n}{\sqrt{\ln n}}
$$

$c|\theta| \leqslant 1$. Кроме того, выполнено неравенство

$$
1-F_{n}(\lambda)<e^{1-\frac{\beta-1}{e \beta} \lambda^{2}}
$$

А. Д. Брюно (Институт прикладной математики им. М. В. Келдыша РАН, г. Москва), Om диофантовых приближений к фундаментальным единицам алгебрачческих полей

Пусть в вещественном $n$-мерном пространстве $\mathbb{R}^{n}=\{X\}$ задано $m$ однородных вещественных форм $f_{i}(X), i=1, \ldots, m, 2 \leqslant m \leqslant n$.

Выпуклая оболочка множества точек $G(X)=\left(\left|f_{1}(X)\right|, \ldots,\left|f_{m}(X)\right|\right)$ для целочисленных $X \in \mathbb{Z}^{n}$ во многих случаях является выпуклым многогранным множеством, граница которого для $\|X\|=O(1)$ вычисляется с помощью стандартной программы.

Точки $X \in \mathbb{Z}^{n}$, для которых значения $G(X)$ лежат на этой границе, названы граничными точками.

Граничные точки $X$ являются наилучшими диофантовыми приближениями для указанных форм. Это дает глобальное обобщение цепной дроби. Для $n=3$ обобщить цепную дробь пытались Л. Эйлер, К. Г. Я. Якоби, П. Г. Л. Дирихле, Ш. Эрмит, Ж. А. Пуанкаре, А. Гурвиц, Ф. Х. Клейн, Г. Минковский, В. Брун, В. И. Арнольд и многие другие.

Пусть $p(\xi)$ - целый неприводимый над $\mathbb{Q}$ многочлен степени $n$, а $\lambda$ - его корень. Оказывается, что набор фундаментальных единиц кольца $\mathbb{Z}[\lambda]$ можно вычислить по граничным точкам некоторой совокупности линейных и квадратичных форм, построенных по корням многочлена $p(\xi)$.

Аналогично вычисляется набор фундаментальных единиц поля $\mathbb{Q}(\lambda)$. До сих пор эти единицы вычислялись только для $n=2$ (с помощью обычных цепных дробей) и $n=3$ (с помощью алгоритма Вороного).

Подход докладчика обобщает цепную дробь, дает наилучшие совместные приближения и основные единицы алгебраических полей для любого $n$.

А. Н. Васильев (Казахстанский филиал Московского государственного университета имени М. В. Ломоносова, г. Астана), Сбалансированные разложения (Доклад основан на совместной работе с А.А. Клячко)

Пусть $F$ - поле и $a \in F$. Будем говорить, что а допускает в $F$ сбалансированное разложение с $k$ сомножителями, если существуют такие $x_{1}, \ldots, x_{k} \in F$, что $x_{1} \cdots x_{k}=a$ и $x_{1}+\cdots+$ $x_{k}=0$. Можно задаться вопросом: для каких $k$ любой элемент данного поля $F$ допускает сбалансированное разложение с $k$ сомножителями? Оказывается, если char $F \neq 2$, то каждый элемент допускает разложение с пятью и более элементами, а именно, если $a \neq 1,0$, то

$$
a=\frac{a}{2} \cdot \frac{a}{2} \cdot(-a) \cdot\left(\frac{2}{a}\right) \cdot\left(-\frac{2}{a}\right)=\frac{1-a}{2} \cdot \frac{1-a}{2} \cdot a \cdot \frac{2}{1-a} \cdot \frac{2}{a-1} \cdot(-1) .
$$

Другие разложения получаются добавлением нужного количества пар $(-1,1)$.

Докладчиком был дан полный ответ на вопрос о сбалансированных разложениях для конечных полей. 
Теорема 1. Пусть $k \geqslant 2$ - целое число и $F$ - конечное поле. Тогда в $F$ каждый элемент допускает сбалансированное разложение с $k$ элементами тогда и только тогда, когда выполнено одно из следующих условий:

- $|F|=2, k$ четно;

- $|F|=4, k \neq 3$;

- количество элементов поля есть степень двойки с показателем не менее $3, k$ произвольное;

- $|F| \in\{3,5\}, k \notin\{2,4\}$;

- $|F|=7, k \notin\{2,3\}$;

- $|F| \notin\{3,5,7\} \cup\left\{2^{n} \mid n \geqslant 1\right\}, k \neq 2$.

Следует отдельно отметить, что этот результат для $k=3$ опирается на оценку Хассе для количества точек на эллиптической кривой.

Можно обратить внимание на то, что разложения на пять и более элементов, упомянутые выше, эквивалентны разложимости с пятью и более сомножителями для элемента $t \in F(t)$. Оказывается, для трех сомножителей такой "универсальной формулы" не существует, т.е. для любого поля $F$ элемент $t \in F(t)$ не допускает сбалансированного разложения с тремя сомножителями.

Доказательство этого факта использует теорему Мэйсона-Стотерса: если три многочлена $x, y, z \in F[t]$ взаимно просты, и выполнено условие $x+y+z=0$, то степень каждого из них не превосходит количества корней произведения $x y z$ в алгебраическом замыкании $F$ либо

$$
\frac{\partial x}{\partial t}=\frac{\partial y}{\partial t}=\frac{\partial z}{\partial t}=0
$$

Имеют смысл также вопросы о сбалансированных разложениях в алгебрах. Будем называть сбалансированное разложение нестепенным, если в нем есть хотя бы два различных элемента. Нестепенную разложимость алгебры наследуют у полей.

Теорема 2. Пусть $F-$ поле, $\operatorname{char} F \neq 2$. Если во всех конечных расширениях $F$ каждый элемент допускает нестепенное сбалансированное разложение с $k$ сомножителями, то это же верно для всякой конечномерной ассощиативной алгебры с единищей над $F$.

СЛЕДСТВИЕ 1. Каждый элемент конечномерной ассоциативной алгебры с единицей над полем $F$ допускает сбалансированное разложение с тремя элементами, если $F$ алгебраически замкнуто, и с пятью элементами, если char $F \neq 2$.

Некоторые вопросы о сбалансированных разложениях остаются открытыми. Особенно стоило бы отметить следующие два из них:

- правда ли, что всякий элемент любого поля раскладывается в произведение не более чем четырех элементов с суммой 0 ?

- допускает ли $t \in \mathbb{C}(t)$ сбалансированное разложение с четырьмя элементами?

Э. Весалаинен (Университет Аалто), Суммы с коэфбициентами Фуръе автоморфньх форм

Пусть $\mathcal{H}$ - верхняя полуплоскость с координатами $z=x+i y$ и мерой $d \mu=d x d y / y^{2}$. На $\mathcal{H}$ имеются лапласиан

$$
-\Delta_{H}=-y^{2}\left(\frac{\partial^{2}}{\partial x^{2}}+\frac{\partial^{2}}{\partial y^{2}}\right)
$$

и действие

$$
\mathrm{SL}(2, \mathbb{R}): z \mapsto \frac{a z+b}{c z+d}
$$

Если ограничиться действием $\mathrm{SL}(2, \mathbb{Z})$, то $L^{2}$ - пространство на факторпространстве относительно инвариантной меры - будет раскладываться в сумму трех подпространств следующим 
каноническим образом:

$$
L^{2}(\mathcal{H} / \mathrm{SL}(2, \mathbb{Z}), d \mu)=\mathbb{C} 1 \oplus\{\text { дискретная часть }\} \oplus\{\text { непрерывная часть }\} .
$$

Мы будем интересоваться дискретной частью, которая состоит из форм Маасса. Всякая форма Маасса $f(x, y)$ имеет разложение в ряд с экспонентами и модифицированными функциями Бесселя следующего вида:

$$
f(x, y)=\sum_{n \neq 0} t(n) \sqrt{y} K_{i \sqrt{\lambda-1 / 4}}(2 \pi|n| y) e(n x),
$$

где $e(x):=e^{2 \pi i x}$. Числа $t(n)$ называются коэфбициентами формы Маaсcа.

Гипотеза Рамануджана-Петерссона для форм Маасса не доказана, поэтому будем обозначать $\vartheta$ такое наименьшее число, что $t(n) \ll n^{\vartheta+\varepsilon}$. Наилучшие на данный момент оценки для $\vartheta$ гласят, что $\vartheta \leqslant 7 / 64$.

Нас будут интересовать оценки вида

$$
\sum_{n \in\left[M, M+M^{\gamma}\right]} a(n) e(n \alpha) \ll M^{\beta+\varepsilon},
$$

где $a(n)$ - коэффициенты формы Маасса или модулярной формы.

Для модулярных форм известны две "тривиальные оценки": $\beta \leqslant 1 / 2$ и $\beta \leqslant \gamma$.

Кроме того, известно, что $\beta \geqslant \gamma / 2$.

Имеются также нетривиальные результаты при $\gamma \in[0.4 ; 0.75]$.

Также для модулярных форм выполнены неравенства

$$
\begin{gathered}
\sum_{n \leqslant M} a(n) \ll M^{1 / 3+\varepsilon}, \\
\sum_{n \leqslant M} a(n) e\left(\frac{n h}{k}\right) \ll k^{2 / 3} M^{1 / 3+\varepsilon} .
\end{gathered}
$$

Аналогичная оценка для форм Маасса выглядит так:

$$
\sum_{n \leqslant M} t(n) e\left(\frac{n h}{k}\right) \ll k^{2 / 3} M^{1 / 3+\vartheta / 3+\varepsilon} .
$$

Кроме того, выполнен аналог оценки Уилтона-Ютилы квадратного корня:

$$
\sum_{n \leqslant M} t(n) e(\alpha n) \ll \sqrt{M}
$$

Подобные вопросы можно решать в случае GL( $n)$-форм Маасса. У GL $(n)$-форм Маасса также бывают коэффициенты Фурье, и мы будем интересоваться оценками вида

$$
\sum_{M \leqslant m \leqslant M+M^{\gamma}} \mathcal{A}(m, 1, \ldots, 1) e(m x) \ll M^{\beta+\varepsilon} .
$$

Оказывается, в общем случае не получается доказать ничего, кроме $\beta \leqslant 1$.

Эта оценка была улучшена Миллером в случае GL(3), а именно он доказал, что можно брать $\beta \leqslant 3 / 4$.

Если же в GL(3)-случае выполнена гипотеза Рамануджана-Петерссона, то можно доказать такой факт:

$$
\sum_{m \leqslant M} \mathcal{A}(n, 1) e\left(\frac{m h}{k}\right) \ll k^{3 / 4} M^{1 / 2+\varepsilon} .
$$


Для очень специального выбора фазы справедливо следующее утверждение.

Теорема 1. Если $w(x)$ - достаточно хорошая функиия, $d, M$ - натуральнье числа, то для всех $\Delta \gg M^{1-1 / n+о(1)}$ выполнено равенство

$$
\begin{aligned}
& \sum_{M \leqslant n \leqslant M+\Delta} \mathcal{A}(m, 1, \ldots, 1) e\left(\frac{d^{1 / n} m}{M^{1-1 / n}}\right) w(m) \\
& =c_{n} d^{1 /(2 n)-1 / 2} \overline{\mathcal{A}(d, 1, \ldots, 1)} \int_{M}^{M+\Delta} w(x) x^{1 /(2 n)-1 / 2} e\left(\frac{d^{1 / n} x}{M^{1-1 / n}}-n x^{1 / n} d^{1 / n}\right) d x \\
& \quad+O\left(\Delta M^{-1 / 2-1 /(2 n)}\right) .
\end{aligned}
$$

Теорема 1 также имеет интересное следствие о возможности восстановить форму Маасса по коэффициентам.

СледствиЕ 1. Пусть $M_{l}$ - любая бесконечно возрастающая последовательность. Если у двух $\mathrm{GL}(n)$-форм совпадают все коэффициенты $\mathcal{A}(m, 1, \ldots, 1)$ с $m \in\left[M_{l}, M_{l}+M_{l}^{\alpha}\right]$, где $\alpha>1-1 / n$, то эти форми совпадают.

С. Б. Гашков, И. С. Сергеев (Московский государственный университет имени М. В. Ломоносова, механико-математический факультет), Об арифметической сложности вычисления некоторых линейных преобразований

Пусть $A$ - некоторая матрица $n \times m$ с вещественными коэффициентами. Она задает оператор $A: \mathbb{R}^{n} \rightarrow \mathbb{R}^{m}$. Предположим, что мы выбрали некоторый набор $B$ линейных функционалов $\mathbb{R}^{k} \rightarrow \mathbb{R}$ ( $k$ может быть разным для разных функционалов). Содержательной задачей является вопрос оценок наименьшего количества функционалов из нашего множества, которым надо воспользоваться, чтобы применить к вектору $x \in \mathbb{R}^{n}$ оператор $A$. Максимальное значение этого количества по всем векторам из $\mathbb{R}^{n}$ называется сложностъю $A$ относительно $B$ и обозначается $L_{B}(A)$.

Наборы, которые появлялись в этом докладе, как правило, состоят из бинарных и унарных операций, а именно:

- набор $\{ \pm\}-(x, y) \rightarrow x+y$ и $(x, y) \rightarrow x-y$;

- $B_{C}$ для положительного $C:\{ \pm\} \cup\{x \rightarrow a x|| a \mid \leqslant C\}$;

- $B_{\infty}-\{(x, y) \rightarrow a x+b y \mid a, b \in \mathbb{R}\}$;

- $B_{>0}-\left\{(x, y) \rightarrow a x+b y \mid a, b \in \mathbb{R}_{\geqslant 0}\right\}$.

Сложность удовлетворяет некоторым естественным свойствам, например:

- $L_{B}(A C) \leqslant L_{B}(A)+L_{B}(C)$;

- если $C$ - подматрица в $A$, то $L_{B}(A) \geqslant L_{B}(C)$;

- $L_{B}(A \oplus C) \leqslant L_{B}(A)+L_{B}(C)$;

- если $A$ - матрица $n \times m$, то $\left|L_{B}(A)-L_{B}\left(A^{T}\right)\right| \ll n+m$.

Кроме того, в 1973 г. Ж. Моргенштерном для всякого положительного $C$ была доказана следующая оценка:

$$
L_{B_{C}}(A) \geqslant \log _{\max (2, C)}|\operatorname{det} A| \text {. }
$$

Пусть $H_{n}$ - матрица Адамара размера $n \times n$. Тогда $\left|\operatorname{det} H_{n}\right|=n^{n / 2}$. Поэтому оценка Моргенштерна доказывает, что

$$
L_{\{ \pm\}}\left(H_{n}\right) \asymp L_{B_{2}}\left(H_{n}\right)=\Theta(n \ln n) .
$$

Для матрицы Паскаля $C_{n}=\left\{\left(\begin{array}{l}i \\ j\end{array}\right)\right\}_{1 \leqslant i, j \leqslant n}$ из тождества $\left(\begin{array}{c}i+1 \\ j+1\end{array}\right)=\left(\begin{array}{c}i+1 \\ j\end{array}\right)+\left(\begin{array}{c}i \\ j\end{array}\right)$ получается оценка $L_{\{ \pm\}}\left(C_{n}\right) \leqslant n^{2} / 2$. Оказывается, однако, что у $C_{n}$ есть подматрица с детерминантом порядка $c^{n^{2}}$, где $c>1$. Таким образом,

$$
L_{B_{2}}\left(C_{n}\right)=\Theta\left(n^{2}\right)
$$


С другой стороны, $C_{n}=\Delta A \Delta^{-1}$, где $\Delta=\operatorname{diag}(0 !, 1 !, \ldots,(n-1) !)$ и $A=\{1 /(i-j) !\}$ (будем считать, что $1 /(-k) !=0$ при $k>0)$, поэтому $L_{B_{\infty}}\left(C_{n}\right) \ll n \ln n$.

Пусть теперь $D_{n}-$ матрица Серпинского, т.е. $D_{n}=C_{n}(\bmod 2)$. Тогда по теореме С. Н. Селезнёвой и Дж. Бояр, М. Г. Файнда (2012) имеем

$$
L_{\{ \pm\}}\left(D_{n}\right) \sim L_{B_{2}}\left(D_{n}\right) \sim \frac{1}{2} n \log _{2} n .
$$

Кроме того,

$$
L_{B>0}\left(D_{n}\right)=\Omega(n \ln n) .
$$

Положим $s_{n}=\left\{s_{i}^{j}\right\}$ и $S_{n}=\left\{S_{i}^{j}\right\}$, где $s$ и $S$ - числа Стирлинга первого и второго рода соответственно. Хорошо известно, что $s_{n} S_{n}=1$. Оказывается, что матрицы $s_{n}$ и $\left|s_{n}\right|$ имеют подматрицы с детерминантами порядка $e^{\Theta\left(n^{2} \ln n\right)}$. Таким образом,

$$
\begin{aligned}
L_{B_{2}}\left(s_{n}\right) & \asymp L_{\{ \pm\}}\left(s_{n}\right)=\Theta\left(n^{2} \ln n\right), \\
L_{B_{2}}\left(\left|s_{n}\right|\right) & \asymp L_{\{ \pm\}}\left(\left|s_{n}\right|\right)=\Theta\left(n^{2} \ln n\right) .
\end{aligned}
$$

Если $V_{n}=\left\{i^{j}\right\}$ - матрица Вандермонда, то $V_{n}=C_{n} \Delta S_{n}^{T}$, поэтому

$$
L_{B_{2}}\left(V_{n}\right) \asymp L_{\{ \pm\}}\left(V_{n}\right)=\Theta\left(n^{2} \ln n\right) .
$$

Кроме того,

$$
L_{B_{\infty}}\left(V_{n}\right), L_{B_{\infty}}\left(S_{n}\right), L_{B_{\infty}}\left(s_{n}\right) \ll n \ln ^{2} n .
$$

Также докладчиками был получен ряд результатов о сложностях вычисления матриц более арифметрического происхождения.

В 2015 г. докладчиками были доказаны следующие две теоремы.

Теорема 1 (С. Б. Гашков, И. С. Сергеев, 2015). Пустъ $\operatorname{GCD}(n)=\{\operatorname{HOД}(i, j)\}_{1 \leqslant i, j \leqslant n} u$ $\varphi(D)=\operatorname{diag}(\varphi(1), \varphi(2), \ldots, \varphi(n))$. Тогда выполнены оценки

$$
L_{B_{2}}(\operatorname{GCD}(n)) \sim L_{\{ \pm\}}(\operatorname{GCD}(n)) \sim n \log _{2} n
$$

$u$

$$
\left|L_{\{ \pm\}}(\operatorname{GCD}(n))-L_{\{ \pm\}}(\varphi(D))\right|=o(n \sqrt{\ln n}) .
$$

И аналогичное утверждение для матрицы из НОКов.

Теорема 2 (С. Б. Гашков, И. С. Сергеев, 2015). $\operatorname{Ecлu~} \operatorname{LCM}(n)=\{\operatorname{HOK}(i, j)\}_{1 \leqslant i, j \leqslant n}, m o$ справедлива асимптотика

$$
L_{B_{2}}(\operatorname{LCM}(n)) \sim L_{\{ \pm\}}(\operatorname{LCM}(n)) \sim 2 n \log _{2} n .
$$

О. Н. Герман (Московский государственный университет имени М. В. Ломоносова, механико-математический факультет), Циклические палиндромы и периодические цепные дроби

Хорошо известно, что если $r>1$ - рациональное число, не являющееся квадратом, то $\sqrt{r}$ раскладывается в цепную дробь вида

$$
\sqrt{r}=\left[a_{0} ; \overline{a_{1}, a_{2}, \ldots, a_{2}, a_{1}, 2 a_{0}}\right] .
$$

Период этой дроби, прочитанный слева направо, также является периодом. Будем называть такие периоды ииклично палиндромическими.

Докладчиком при помощи геометрии квадратичных иррациональностей был доказан следующий критерий цикличной палиндромичности периода. 
ТеОрема 1. Квадратичная иррациональность а имеет ииклично палиндромический период цепной дроби, если и только если существует такое $\omega \sim \alpha$, что выполнено одно из четырех условий:

1) $\omega+\bar{\omega}=0$;

2) $\omega+\bar{\omega}=1$;

3) $\omega \bar{\omega}=1$;

4) $\omega \bar{\omega}=-1$.

Здесь $x \sim y$ означает, что существует матрица $\gamma \in \mathrm{SL}(2, \mathbb{Z})$ с $\gamma x=y$, a $\bar{\omega}$ - сопряљенное $\kappa \omega$ число. Кроме того, существование $\omega$ с условием 2) эквивалентно существованию $\omega$ с условием 3).

Доказательство построено на рассмотрении многоугольников Клейна.

Пусть $\alpha$ - иррациональное число. Будем обозначать $\mathcal{L}_{\alpha}$ прямую в $\mathbb{R}^{2}$, проходящую через точки $(0,0)$ и $(1, \alpha)$. Для двух различных иррациональных вещественных чисел $\alpha$ и $\beta$ прямые $\mathcal{L}_{\alpha}$ и $\mathcal{L}_{\beta}$ разбивают плоскость на четыре угла. Для каждого из углов рассмотрим выпуклую оболочку всех целочисленных точек, лежащих внутри этого угла. Эти четыре (обобщенных) многоугольника называются многоуголъниками Клейна. Два многоугольника Клейна называются смежными, если они соответствуют смежным углам.

Будем говорить, что отрезок с целочисленными концами $n y c m$, если в его внутренности нет целых точек. Целочисленной длиной отрезка называется количество пустых отрезков внутри него. Целочисленным углом между двумя пустыми отрезками, имеющими один общий конец, мы называем площадь параллелограмма, натянутого на эти отрезки.

Теорема 1 следует из того, что число $\alpha$ имеет циклично палиндромический период цепной дроби тогда и только тогда, когда многоугольник Клейна, построенный на $\mathcal{L}_{\alpha}$ и $\mathcal{L}_{\bar{\alpha}}$, имеет некоторую специальную симметрию.

Также существенную роль в доказательстве играет следующая лемма, доказанная Е. Коркиной.

ЛЕмма 1 (Е.И. Коркина, 1995). Пусть $\left(a_{k}\right)_{k \in \mathbb{Z}}$ - любая последовательность натуральных чисел, а $\left[v_{-2}, v_{0}\right]$ - любой челочисленный отрезок челочисленной длины $a_{0}$. Предположим, что все челье точки, которые ближе $к$ прямой $v_{-2} v_{0}$, чем точка $(0,0)$, лежат на этой прямой. Тогда существует единственный многоугольник Клейна $\mathcal{K}$ с вершинами $v_{2 m}$, $m \in \mathbb{Z}$, такой, что для всякого иелого $m$ выполнено:

- $\left[v_{2 m-2}, v_{2 m}\right]-$ ребро $\mathcal{K}$;

- челочисленная длина этого ребра равна $a_{2 m}$;

- челочисленный угол при вершине $v_{2 m}$ равен $a_{2 m+1}$.

С. А. Гриценко (Московский государственный университет имени М. В. Ломоносова, механико-математический факультет), О нулях функиии Дэвенпорта-Хейльбронна, лежащих на критической прямой

Хорошо известна теорема Атле Сельберга о том, что положительная доля всех нулей дзета-функции Римана лежит на критической прямой. В доказательстве этой теоремы существенно использовались так называемые "успокаивающие множители”, строящиеся по эйлеровому произведению для $\zeta(s)$. Однако для многих рядов Дирихле без эйлерового произведения, но с функциональным уравнением доказано, что аномально много нулей лежит на критической прямой. Простейший пример такого ряда Дирихле - функция Дэвенпорта-Хейльбронна

$$
f(s)=\frac{1-i \kappa}{2} L\left(s, \chi_{1}\right)+\frac{1+i \kappa}{2} L\left(s, \bar{\chi}_{1}\right)
$$


где

$$
\kappa=\frac{\sqrt{10-2 \sqrt{5}}-2}{\sqrt{5}-1},
$$

$\chi_{1}$ - характер Дирихле по модулю 5 с $\chi_{1}(2)=i$.

Пусть $N(\sigma, T, f)$ - число нулей $f$ с $\operatorname{Re} s \geqslant \sigma$ и $0<\operatorname{Im} s \leqslant T$. До 1980 г. было известно только то, что $N(1, T, f) \gg T$ и $N(a, T, f)-N(b, T, f) \asymp T$ при $1 / 2<a<b \leqslant 1$.

В 1980 г. С. М. Воронин доказал, что

$$
N_{0}(T, f)>T e^{(1 / 20) \sqrt{\ln \ln \ln \ln T}},
$$

где $N_{0}(T, f)$ - количество нулей на критической прямой с $|\operatorname{Im} s|<T$.

В 1989 г. А.А. Карацуба разработал метод, позволяющий использовать множитель, частично успокаивающий оба слагаемых в функции $f(s)$, и доказать, что

$$
N_{0}(T, f)>T(\ln T)^{1 / 2-\varepsilon} \text {. }
$$

Величина 1/2 в показателе степени возникает из-за того, что плотность простых в совпадающих частях эйлеровых произведений для слагаемых равна $1 / 2$.

А. А. Карацуба использовал оригинальный метод, заключающийся в том, что некоторые вспомогательные функции в доказательстве возводятся в очень маленькую положительную степень. Докладчик внес в метод А. А. Карацубы ряд существенных изменений. А именно, он использовал множитель, который по-разному "успокаивает" слагаемые $L\left(s, \chi_{1}\right)$ и $L\left(s, \overline{\chi_{1}}\right)$ : одно - чуть сильнее, другое - чуть слабее. Грубо говоря, это дает возможность работать только с одним “доминирующим" слагаемым. Кроме того, автор использовал технику, позволяющую получать весьма точные нижние оценки для дробных моментов рядов Дирихле на критической прямой. Применение соображений такого рода позволило докладчику получить следующий результат.

Теорема 1. Для всякого $\varepsilon>0$ имеет место нижняя оценка

$$
N_{0}(2 T, f)-N_{0}(T, f) \gg_{\varepsilon} T(\ln T)^{9 / 16-\varepsilon} .
$$

"Предел" этого метода описывается таким условным результатом.

Теорема 2. Если выполнена гипотеза Римана для $L\left(s, \chi_{1}\right)$, то для всякого $\varepsilon>0$ выполнена оценка

$$
N_{0}(T, f) \gg_{\varepsilon} T(\ln T)^{5 / 8-\varepsilon} \text {. }
$$

И.Ш. Джаббаров (Гянджинский государственный университет), Влияние особенностей в задаче о показателе сходимости многомерной проблемь Терри

Пусть $P \geqslant 2, n \geqslant 2$ и $J_{k, n}(P)$ - число решений системы уравнений

$$
x_{1}^{i}+\cdots+x_{k}^{i}=x_{k+1}^{i}+\cdots+x_{2 k}^{i} \quad \text { при } \quad 1 \leqslant i \leqslant n
$$

в целых числах $1 \leqslant x_{1}, \ldots, x_{2 k} \leqslant P$. В решении многих задач теории чисел большую роль играют верхние оценки величины $J_{k, n}(P)$. Например, оценки такого рода, полученные И. М. Виноградовым, были использованы им для нахождения наилучшей на сегодняшний день границы области критической полосы $0<\operatorname{Re} s<1$, свободной от нулей $\zeta(s)$, и для новой оценки остаточного члена в асимптотическом законе распределения простых чисел.

Можно также ставить вопрос об асимптотике $J_{k, n}(P)$. В 1938 г. Хуа Ло-Кеном при помощи метода Виноградова была доказана следующая асимптотическая формула. 
Теорема 1. Для некоторых положительных $\delta$ и с при $k>c n^{2} \ln n$ имеет место равенство

$$
J_{k, n}(P)=\sigma \theta_{0} P^{2 k-n(n+1) / 2}+O\left(P^{2 k-n(n+1) / 2-\delta}\right),
$$

где $\sigma$ и $\theta_{0}-$ так называемые особый ряд и особый интеграл этой задачи.

Также Хуа Ло-Кеном было доказано, что интеграл сходится при $2 k>n^{2}+n / 2$. В связи с этим был поставлен вопрос о показателе сходимости этого особого интеграла, т.е. о таком значении $\gamma$, что при $2 k>\gamma$ интеграл $\theta_{0}$ сходится, а для $2 k<\gamma-$ расходится.

В общем случае вопрос можно поставить следующим образом: пусть $r \geqslant 1, N \geqslant 2$ - целые числа, $\gamma_{j}(\bar{x})=x_{1}^{k_{1 j}} \cdots x_{r}^{k_{r j}}-$ набор $N$ мономов от $r$ переменных. Определим

$$
F(\bar{x})=\sum_{j=1}^{N} \alpha_{j} \gamma_{j}(\bar{x})
$$

Найти показатель сходимости интеграла

$$
\theta_{k}=\int_{\mathbb{R}^{N}}\left|\int_{[0,1]^{k}} e^{2 \pi i F(\bar{x})} d \bar{x}\right|^{2 k} d \alpha_{1} \ldots d \alpha_{N} .
$$

Одномерный случай этой задачи для мономов $x, x^{2}, \ldots, x^{n}$ впервые решен Г. И. Архиповым, А.А. Карацубой и В.Н. Чубариковым. Они доказали, что показатель сходимости равен $n(n+1) / 2+1$. В “выщербленном" случае (т.е. для мономов $x^{n_{1}}, \ldots, x^{n_{k}}$ с условиями $n_{1}<n_{2}<\cdots<n_{k}$ и $\left.n_{1}+\cdots+n_{k}<\left(n_{k}+1\right) n_{k} / 2\right)$ ответ теми же авторами получен немного другой, а именно,

$$
\gamma=n_{1}+\cdots+n_{k}
$$

В многомерном же случае имеются верхние оценки, однако вопрос о точном значении остается открытым.

При исследовании этой задачи докладчиком была получена формула для $\theta_{k}$ в виде некоторого поверхностного интеграла.

Теорема 2 (И.Ш. Джаббаров). Пусть П - подмножество в $[0,1]^{k r}=\left([0,1]^{k}\right)^{r}$, заданное системой уравнений

$$
\gamma_{j}\left(\bar{x}_{1}\right)+\cdots+\gamma_{j}\left(\bar{x}_{k}\right)=\gamma_{j}\left(\bar{x}_{k+1}\right)+\cdots+\gamma_{j}\left(\bar{x}_{2 k}\right) \quad \text { npu } \quad 1 \leqslant j \leqslant N .
$$

Тогда справедливо равенство

$$
\theta_{k}=(2 \pi)^{N} \int_{\Pi} \frac{d s}{\sqrt{G}},
$$

где $G$ - определитель Грама градиентов функиий

$$
f_{j}=\gamma_{j}\left(\bar{x}_{1}\right)+\cdots+\gamma_{j}\left(\bar{x}_{k}\right)-\gamma_{j}\left(\bar{x}_{k+1}\right)-\cdots-\gamma_{j}\left(\bar{x}_{2 k}\right), \quad j=1, \ldots, N .
$$

При этом сходимость левой части равенства эквивалентна сходимости правой.

При помощи этой теоремы удается доказать следующую оценку.

Теорема 3. Пусть $\rho$-ранг матриць $\left\{k_{i j}\right\}$ показателей мономов. Если $k \rho \geqslant N u$

$$
2 k r>r+\sum_{i, j} k_{i j},
$$

то интеграл $\theta_{k}$ сходится. 
Доказательство также существенно использует тот факт, что алгебраическое множество П, заданное в $\mathbb{R}^{k r}$ системой уравнений

$$
\gamma_{j}\left(\bar{x}_{1}\right)+\cdots+\gamma_{j}\left(\bar{x}_{k}\right)=\gamma_{j}\left(\bar{x}_{k+1}\right)+\cdots+\gamma_{j}\left(\bar{x}_{2 k}\right) \quad \text { при } \quad 1 \leqslant j \leqslant N,
$$

является алгебраическим многообразием специального вида.

Н. М. Добровольский (Тульский государственный педагогический университет им. Л. Н. Толстого), О новых результатах в теории гиперболической дзета-функиии решеток

Пусть $\Lambda$ - некоторая решетка в $\mathbb{R}^{s}$. В связи с различными вопросами из теории решеток хотелось бы рассматривать для произвольной решетки ее дзета-функцию

$$
\zeta(\Lambda \mid \alpha)=\sum_{\substack{x \in \Lambda \\ x_{i} \neq 0}} \frac{1}{\left|x_{1} \cdots x_{s}\right|^{\alpha}}
$$

Однако оказывается, что во многих содержательных примерах этот ряд не сходится ни для какого $\alpha$. Заменой обыкновенной дзета-функции в этом случае может служить гиперболическая дзета-функиия решетки $\Lambda$. Определяется она следующим образом:

$$
\zeta_{H}(\Lambda \mid \alpha)=\sum_{\substack{x \in \Lambda \\ x \neq 0}} \frac{1}{\left(\overline{x_{1}} \ldots \overline{x_{s}}\right)^{\alpha}}
$$

где $\bar{x}=\max (1,|x|)$.

Если для целочисленной решетки $\Lambda$ ряд для обыкновенной дзета-функции сходится, то она удовлетворяет функциональному уравнению.

ТЕОрема 1. Пусть $\Lambda$ - иелочисленная s-мерная решетка со сходящимся дзета-рядом, $\operatorname{det} \Lambda=N$, a $\Lambda^{*}-$ двойственная решетка. Тогда выполнено равенство

$$
\zeta(\Lambda \mid \alpha)=\frac{M(\alpha)^{s}}{N} \zeta\left(\Lambda^{*} \mid 1-\alpha\right), \quad \text { əде } \quad M(\alpha)=\frac{2 \Gamma(1-\alpha)}{(2 \pi)^{1-\alpha}} \sin \frac{\pi \alpha}{2} .
$$

Возможно, гиперболическая дзета-функция также во многих случаях удовлетворяет функциональному уравнению, однако, оно должно быть сложнее, поскольку, например, в случае $\Lambda=d \cdot \mathbb{Z}$ выполнено равенство

$$
\zeta_{H}(\Lambda \mid \alpha)=\zeta(\Lambda \mid \alpha)+f(\alpha, d) .
$$

Здесь $f(\alpha, d)$ - конечный ряд Дирихле следующего вида:

$$
f(\alpha, d)= \begin{cases}0, & \text { если } d \geqslant 1, \\ \sum_{1 \leqslant m \leqslant \frac{1}{d}}\left(1-\frac{1}{(d m)^{\alpha}}\right) & \text { в остальных случаях. }\end{cases}
$$

Один из самых существенных вопросов о гиперболической дзета-функции - ее асимптотическое поведение при растущем детерминанте в различных семействах. Эта задача решена в случае решетки кольца целых вещественного поля.

Теорема 2 (Н. М. Добровольский, В. С. Ванькова, С. Л. Козлова). Пусть $F-$ чисто вещественное числовое поле, $\Lambda(t, F)=t \Lambda(F)$, где $\Lambda(F)$ - решетка геометрической реализачии кольиа иелых поля $F$. Тогда имеет место следующее асимптотическое выражение:

$$
\zeta_{H}(\Lambda(t, F) \mid \alpha)=\frac{\left.2(\operatorname{det} \Lambda(F))^{\alpha}\right)}{R(s-1) !}\left(\sum_{w} \frac{1}{N(w)^{\alpha}}\right) \frac{\ln ^{s-1} \operatorname{det} \Lambda(t, F)}{(\operatorname{det} \Lambda(t, F))^{\alpha}}+O\left(\frac{\ln ^{s-2} \operatorname{det} \Lambda(t, F)}{(\operatorname{det} \Lambda(t, F))^{\alpha}}\right),
$$

где суммирование ведется по главным идеалам, а $R$-регулятор поля $F$. 
Оказывается, в случае квадратичного $F$ асимптотику удается сильно уточнить.

Теорема 3. Пусть $F$ - квадратичное поле, $R$ - его регулятор, $\zeta_{F}(\alpha)$ - дзета-функиия Дедекинда поля F. Тогда выполнено равенство

$$
\begin{aligned}
\zeta_{H}(\Lambda(t, F) \mid \alpha)=\frac{2(\operatorname{det} \Lambda(F))^{\alpha} \zeta_{F}(\alpha)}{R} \frac{\ln \operatorname{det} \Lambda(t, F)}{(\operatorname{det} \Lambda(t, F))^{\alpha}} \\
\quad-\frac{2(\operatorname{det} \Lambda(F))}{R(\operatorname{det} \Lambda(t, F))^{\alpha}}\left(\zeta_{F}(\alpha) \ln \operatorname{det} \Lambda(F)+\zeta_{F}^{\prime}(\alpha)\right) \\
+\frac{2(\operatorname{det} \Lambda(F))^{\alpha} \zeta_{F}(\alpha)}{(\operatorname{det} \Lambda(t, F))^{\alpha}}\left(\theta_{1}(\alpha)+\frac{\theta_{2}(\alpha)}{\operatorname{sh} \alpha R / 2}\right) .
\end{aligned}
$$

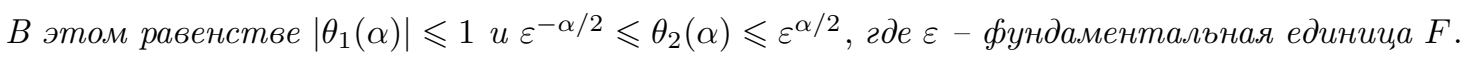

Н. П. Долбилин (Математический институт им. В. А. Стеклова Российской академии наук, г. Москва), Локально антиподалъные множества Делоне

Пусть $G \subset \operatorname{Isom}\left(\mathbb{R}^{d}\right)$ - кристаллографическая группа, т.е. подгруппа с компактной фундаментальной областью. Орбита $G x$ какой-нибудь точки $x \in \mathbb{R}^{d}$ называется регулярной системой. Составляющей частью 18-й проблемы Гильберта является следующая гипотеза.

ГипотезА 1. Любая кристаллографическая группа содержит подгруппу переносов конечного индекса.

Это утверждение было доказано А. М. Шёнфлисом для случая $d=3$, а в остальных размерностях - Л. Бибербахом. Таким образом, всякая регулярная система есть объединение конгруэнтных параллельных друг другу решеток.

Можно также дать эквивалентное определение регулярной системы при помощи следующих трех аксиом.

Теорема 1. Подмножество $X \subset \mathbb{R}^{d}$ - регулярная система, если и толъко если:

(r) существует $r>0$ такое, что $B_{x}(r) \cap B_{y}(r)=\varnothing$ для всех $x, y \in X, x \neq y$;

(R) существует $R>0$ такое, что $\bigcup_{x \in X} B_{x}(R)=\mathbb{R}^{d}$;

$(\mathrm{G})$ множество $X$ выглядит одинаково из любой точки $x \in X$, m.е. группа симметрий множества $X$ действует на него транзитивно.

Если же для множества $X$ выполнены только аксиомы $(r)$ и $(R)$, то $X$ называется множеством Делоне.

Последняя аксиома влечет некоторую локальную регулярность. А именно, для всякого $\rho>0$ будем называть $\rho$-кластером вокруг точки $x \in X$ множество

$$
C_{x}(\rho)=\left\{x^{\prime} \in X:\left|x x^{\prime}\right| \leqslant \rho\right\} .
$$

$\rho$-Кластеры вокруг точек $x$ и $y$ называются эквивалентными, если существует изометрия $g$ такая, что $g(x)=y$ и $g\left(C_{x}(\rho)\right)=C_{y}(\rho)$. Для положительного $\rho$ обозначим через $N(\rho)$ число классов эквивалентности $\rho$-кластеров.

Можно доказать, что $X$ - регулярная система тогда и только тогда, когда $N(\rho) \equiv 1$.

Оказывается, иногда достаточно обращения $N(x)$ в 1 в одной точке.

Теорема 2 (Б. Н. Делоне, Н. П. Долбилин, М. И. Штогрин, Р. В. Галиулин). Множество $X$ - регулярная система тогда и только тогда, когда существует $\rho>0$ такое, что:

1) $N(\rho+2 R)=1$;

2) $S_{x}(\rho)=S_{x}(\rho+2 R)$, где $S_{x}(\rho)$ - группа автоморфизмов $C_{x}(\rho)$. 
Для асимметричных множеств Делоне также справедлива

ТеОРема 3. Если множество Делоне асимметрично, т.е. группа $S_{x}(2 R)$ тривиалъна, и $N(4 R)=1$, то $X-$ регулярная система.

Оказывается, эта оценка в некотором смысле оптимальна.

Теорема 4. Для всякого $\varepsilon>0$ существует нерегулярное множество Делоне $X$ с

$$
N(4 R-\varepsilon)=1
$$

Теорема 5 (Н.П. Долбилин). Для всякого $d>0 u r, R>0$ существует $c=c(d, R / r)$ такая, что если в $(r, R)$-множестве Делоне выполнено $N(c R)=1$, то это множество Делоне - регулярная система.

В маленьких размерностях можно даже избавиться от всяких предположений о структуре нашего множества.

Теорема 6 (М. И. Штогрин, Н. П. Долбилин). Пусть $X \subset \mathbb{R}^{d}-$ множество Делоне. Тогда:

1) если $d=2$ и $N(4 R)=1$, то $X$ регулярно;

2) если $d=3$ и $N(10 R)=1$, то $X$ регулярно.

Будем называть множество Делоне локально антиподальным, если для всех $x \in X 2 R$ кластер вокруг $x$ центрально-симметричен относительно $x$.

Оказывается, локально антиподальное множество Делоне всегда центрально-симметрично. Более того, локальная антиподальность - это очень сильное требование.

Теорема 7 (Н.П. Долбилин, А.Н. Магазинов). Всякое $d$-мерное локально антиподальное множество Делоне есть обгединение $n<2^{d}$ конгруэнтных и параллельных друг другу решеток.

Т. Йонсен (Университет Тромсё), Колъца Стенли-Рейснера, связаннъе с матроидами и кодами

Матроидом, ассоциированным с матрицей $M$ над полем $F$ с $n$ столбцами называется пара $\left(E=\{1, \ldots, n\}, \mathcal{N} \subset 2^{E}\right)$, где подмножество $A \subseteq E$ принадлежит $\mathcal{N}$ тогда и только тогда, когда столбцы с номерами, лежащими в этом подмножестве, линейно независимы. Базисами $\mathcal{B}$ нашего матроида называется набор максимальных подмножеств в $\mathcal{N}$. Набор подмножеств $\mathcal{N}$ удовлетворяет таким аксиомам:

1) $\varnothing \in \mathcal{N}$;

2) если $I \in \mathcal{N}, I^{\prime} \subset I$, то $I^{\prime} \in \mathcal{N}$;

3) если $I_{1}, I_{2} \in \mathcal{N},\left|I_{1}\right|<\left|I_{2}\right|$, то существует $e \in I_{2} \backslash I_{1}$ такое, что $I_{1} \cup\{e\} \in \mathcal{N}$.

Конечный матроид - это пара $M=(E, \mathcal{N})$, где $E$ - конечное множество, а $\mathcal{N} \subset 2^{E}$ удовлетворяет указанным выше аксиомам. Базисами матроида, как и ранее, называются максимальные элементы в $\mathcal{N}$. Элементы $\mathcal{N}$ называются независимыми подмножествами $E$. Очевидно, любой базис имеет один и тот же размер. Он называется рангом матроида. Разумеется, существуют матроиды, которые не являются матроидами никакой матрицы.

Двойственный матроид $M^{*}$ определяется как $\left\{E, \mathcal{N}^{*}\right\}$, где $\mathcal{B}^{*}$ состоит из дополнений до $\mathcal{B}$. Легко доказать также, что

$$
\operatorname{rk}(M)+\operatorname{rk}\left(M^{*}\right)=n=|E| .
$$

Матроид линейного кода $C$ (т.е. подпространства $\left.C \subseteq\left(\mathbb{F}_{q}\right)^{n}\right)$ - это матроид $M(C)$ любой матрицы проверки нашего кода на четность (т.е. матрицы коэффициентов уравнений нашего подпространства). 
Код $C$ называется $[n, n-\operatorname{rk}(M(C))]$-кодом. В этом случае $M(C)^{*}=M\left(C^{*}\right)$, где $C^{*}-$ ортогональное дополнение $C$.

Очень многие инварианты кода на самом деле выражаются в терминах матроидов.

Введем функцию ранга

$$
\operatorname{rk}: 2^{E} \rightarrow \mathbb{Z}_{\geqslant 0}, \quad \text { rk: } X \mapsto \max \left\{\left|X^{\prime}\right|: X^{\prime} \subseteq X, X^{\prime} \in \mathcal{N}\right\} .
$$

Она удовлетворяет таким трем аксиомам:

R1) если $X \subseteq E$, то $0 \leqslant \operatorname{rk}(X) \leqslant|X|$;

R2) если $X \subseteq Y \subseteq E$, то $\operatorname{rk}(X) \leqslant \operatorname{rk}(Y)$;

R3) для всех $X, Y \subseteq E$ выполнено $\operatorname{rk}(X \cup Y)+\operatorname{rk}(X \cap Y) \leqslant \operatorname{rk}(X)+\operatorname{rk}(Y)$.

Цикл в матроиде - это минимальное зависимое (не независимое) множество. Множество всех циклов обозначается $\mathcal{C}(M)$. Ясно, что $\mathcal{C}(M)$ полностью определяет $M$.

Пусть теперь $C \subseteq F^{n}$ для некотрого конечного алфавита $F$ с $|F|=q$. Пусть для всякого $X \subseteq E=\{1, \ldots, n\}$ проекция $C_{X}$ имеет размер $q^{s}$ для некоторого неотрицательного $s(X)$. Такое $C$ называется почти афбфинным кодом.

Если $C$ - почти аффинный код, то $\operatorname{rk}(X)=\log _{q}\left|C_{X}\right|$ определяет функцию ранга некоторого матроида. Тогда $M(C)$ - двойственный к этому матроиду матроид.

Пусть $C$ - линейный код над полем $\mathbb{K}$. Обобщенные веса Хэммингса для $C$ определяются так:

$$
d_{i}=\min \{\# \operatorname{supp} D: D-\text { линейный подкод в } D \text { размерности } i\} \text {. }
$$

Для почти аффинных кодов обобщенные веса Хэммингса определяются аналогичным образом.

Оказывается, для весов Хэммингса можно дать эквивалентное определение в терминах матроидов. Для матроида $N$ на множестве $E$ обобщенные веса Хэммингса равны

$$
d_{i}=\min \{\# \sigma \mid \# \sigma-\operatorname{rk}(\sigma)=i\} .
$$

Далее, если $M$ - матроид на множестве $E$, а $\mathbb{K}$ - поле, определим $S=\mathbb{K}[X]=\mathbb{K}\left[X_{e}, e \in E\right]$. Идеалом Стенли-Рейснера для $M$ называется идеал $I_{M} \subset S$, порожденный $X^{\sigma}=\prod_{e \in \sigma} X_{e}$, где

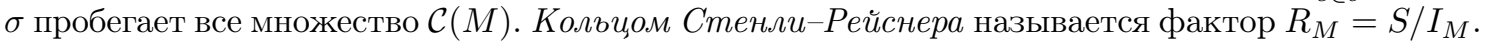

Из минимальной свободной резольвенты для $R_{M}$ получаются три типа чисел Бетти матроида. Если $i$-й член этой резольвенты равен

$$
F_{i}=\bigoplus_{\alpha \in \mathbb{N} E} S(-\alpha)^{\beta_{i, \alpha}}
$$

то $\mathbb{N}^{E}$-градуированные числа Бетти - это $\beta_{i, \alpha} ; \mathbb{N}$-градуированные числа Бетти определяются KaK

$$
\beta_{i, d}=\sum_{|\alpha|=d} \beta_{i, \alpha}
$$

и, наконец, глобальные числа Бетти равны

$$
\beta_{i}=\sum_{d \geqslant 0} \beta_{i, d}
$$

Оказывается, $\mathbb{N}^{E}$-градуированные числа Бетти равны размерностям гомологий соответствующих ограничений матроида как симплициального комплекса.

Кроме того, в терминах чисел Бетти можно выразить и обобщенные веса Хэммингса.

Теорема 1. Пусть $M-$ матроид ранга $r$ на множестве E. Тогда при $1 \leqslant i \leqslant \# E-r$ его обобщенные веса Хэммингса равнь

$$
d_{i}=\min \left\{d \mid \beta_{i, d} \neq 0\right\} .
$$


А. Б. Калмынин (Национальный исследовательский институт «Высшая школа экономики», математический факультет), О числах Новака

Числа Новака - это такие натуральные $n$, что $n \mid 2^{n}+1$. Пусть $N_{B}(x)$ - “считающая функция" множества чисел Новака. Буква " $B$ ” в обозначении считающей функции написана в честь профессора Карлова университета Праги Бржетислава Новака, который доказал первые нетривиальные оценки для $N_{B}(x)$.

В 2012 г. Х. Х. Альба Гонзалез, Ф. Люка, К. Померанц и И.Е. Шпарлинский доказали следующую двойную оценку для этой величины:

$$
e^{c(\ln \ln x)^{2}} \ll N_{B}(x) \ll x e^{-(1+o(1)) \sqrt{\ln x \ln \ln x}} .
$$

Докладчиком были получены более сильные нижние оценки функции $N_{B}(x)$, а именно

ТЕОРема 1. Для любого биксированного натурального числа $n \geqslant 2$ существует такая константа $c_{n}>0$, что выполнена оценка

$$
N_{B}(x) \gg e_{n}\left(c_{n}\left(\ln _{n+1} x\right)^{2}\right),
$$

где $e_{n}-n$-кратная экспонента, $a \ln _{n+1}-(n+1)$-кратный логарифф.

Та же оценка выполнена и для последовательности таких натуральных $n$, что $n \mid a^{n}-b^{n}$, где $a$ и $b$ - целые числа с условием $|a-b|>1$.

Ясно, что не всякое простое число может делить какое-нибудь число Новака. Будем называть такие простые $p$, что существует число Новака $N$, делящееся на $p$, простыми числами Новака. Из обобщенной гипотезы Римана можно вывести следующую оценку на их считающую функцию.

Теорема 2. Пусть $\pi_{N}(x)$ - количество простых чисел Новака, не превосходящих х. Если верна обобщенная гипотеза Римана, то

$$
\pi_{N}(x) \ll \frac{x \sqrt{\ln \ln x}}{(\ln x)^{3 / 2}} .
$$

Числами Новака-Кармайкла назовем такие $n$, что для всякого $a$, взаимно простого с $n$, число $a^{n}-1$ делится на $n$. Оказывается, простые делители таких чисел Новака-Кармайкла вида $2 N$, что $N$ - число Новака, имеют следующее простое описание.

Теорема 3. Пусть $P_{1}$ - множество всех простых чисел, сравнимых с 3 по модулю 8. Для всех $n>0$ определим $P_{n+1}$ как множество всех простых чисел $p \in P_{n}$ таких, что все простые делители числа $(p-1) / 2$ также лежат в $P_{n}$. Пусть

$$
P_{\infty}=\bigcap_{n=1}^{+\infty} P_{n} .
$$

Тогда простое число р является делителем числа Новака $N$ такого, что $2 N$ - число Новака-Кармайкла, если и только если оно лежит в $P_{\infty}$.

В связи с этим была высказана такая гипотеза.

ГиПотезА 1. Множество $P_{\infty}$ бесконечно.

На данный момент не известно даже, бесконечны ли множества $P_{n}$ для всех натуральных $n$. Д. В. Коледа (Институт математики НАН Беларуси), Совместное распределение вещественных сопряженных алгебрачческих чисел

Хорошо известно, что рациональные числа со знаменателем, не превосходящим $Q$, на любом отрезке при $Q \rightarrow+\infty$ распределяются равномерно. 
Возникает естественный вопрос: как распределены алгебраические числа заданной степени $n \geqslant 2$ с высотой не больше $Q$, когда $Q$ неограниченно возрастает?

Здесь высотой $H(\alpha)$ алгебраического числа $\alpha$ называется максимум модулей коэффициентов многочлена наименьшей степени с целыми взаимно простыми коэффициентами, для которого $\alpha$ является корнем, а степенъю $\operatorname{deg} \alpha$ - соответственно степень этого многочлена.

В 2012 г. докладчик доказал такую теорему.

Теорема 1 (Коледа, 2012). Пусть $I \subset \mathbb{R}$ - некоторьй интервал. Пусть $\Phi(Q ; I)-$ количество алгебраических чисел $\alpha \in I$ степени $\operatorname{deg} \alpha \leqslant n$ и высоты $H(\alpha) \leqslant Q$. Тогда при $Q \rightarrow+\infty$ справедливо

$$
\Phi(Q ; I)=\frac{Q^{n+1}}{2 \zeta(n+1)} \int_{I} \varphi_{n}(x) d x+O\left(Q^{n} D\right),
$$

где положительная функиия $\varphi_{n}(x)$ имеет вид

$$
\varphi_{n}(x)=\int_{B_{n}(x)}\left|\sum_{k=1}^{n} k t_{k} x^{k-1}\right| d t_{1} \ldots d t_{n},
$$

гдe

$$
B_{n}(x)=\left\{\left(t_{1}, \ldots, t_{n}\right) \in \mathbb{R}^{n}: \max _{1 \leqslant k \leqslant n}\left|t_{k}\right| \leqslant 1,\left|\sum_{k=1}^{n} t_{k} x^{k}\right| \leqslant 1\right\} .
$$

Неявная постоянная в символе $O(\cdot)$ зависит только от $n ; u$

$$
D= \begin{cases}\ln Q, & \text { если } n=2 \\ 1, & \text { если } n>2\end{cases}
$$

При малых $x$ функцию плотности $\varphi_{n}(x)$ можно выписать явно, а именно, при $|x| \leqslant x_{n}$, где $x_{n}>1-1 / \sqrt{2}$ - некоторая зависящая от $n$ постоянная, выполнено равенство

$$
\varphi_{n}(x)=\frac{2^{n-1}}{3}\left(3+\sum_{k=1}^{n-1}(k+1)^{2} x^{2 k}\right)
$$

Любопытно, что $\varphi_{n}(x)$ совпадает (с точностью до постоянного множителя) с плотностью вещественных корней случайного многочлена с независимыми равномерно распределенными на $[-1,1]$ коэффициентами.

Посмотрим теперь на совместное распределение сопряженных вещественных алгебраических чисел.

Теорема 2 (Гётце, Коледа, Запорожец, 2015). Пусть $B \subset \mathbb{R}^{k}$ - тело, ограниченное $m$ алгебраическими гиперповерхностями степени $\leqslant d$. Пусть $\Phi_{k}(Q ; B)$ - количество векторов в $B$, компоненты которых являются сопряженными вещественными алгебраическими числами степени $n$ и высоть $\leqslant Q$. Тогда имеет место равенство

$$
\Phi_{k}(Q ; B)=\frac{(2 Q)^{n+1}}{2 \zeta(n+1)} \int_{B} \rho_{k}(\mathbf{x}) d \mathbf{x}+O\left(Q^{n} D\right),
$$

где $d \mathbf{x}=d x_{1} d x_{2} \ldots d x_{k}$, неявная постоянная в символе $O(\cdot)$ зависит только от $n, m u d$; неотрицательная функция $\rho_{k}: \mathbb{R}^{k} \rightarrow \mathbb{R}$ задана формулой

$$
\rho_{k}(\mathbf{x})=2^{-n-1} \prod_{1 \leqslant i<j \leqslant k}\left|x_{i}-x_{j}\right| \int_{D_{\mathbf{x}}} \prod_{i=1}^{k}\left|\sum_{j=0}^{n-k} t_{j} x_{i}^{j}\right| d t_{0} \ldots d t_{n-k},
$$


где

$$
D_{\mathbf{x}}=\left\{\left(t_{0}, \ldots, t_{n-k}\right) \in \mathbb{R}^{n-k+1}: \max _{0 \leqslant i \leqslant n}\left|\sum_{j=0}^{n-k}(-1)^{j} \sigma_{k-i+j}(\mathbf{x}) t_{j}\right| \leqslant 1\right\},
$$

здесъ $\sigma_{s}(\mathbf{x})$ - элементарный симметричный многочлен степени $s$ :

$$
\sigma_{s}(\mathbf{x}):= \begin{cases}1 & \text { для } s=0, \\ \sum_{1 \leqslant j_{1}<\cdots<j_{s} \leqslant k} x_{j_{1}} x_{j_{2}} \cdots x_{j_{s}} & \text { для } 1 \leqslant s \leqslant k, \\ 0 & \text { иначе. }\end{cases}
$$

Следует отметить, что предельное равенство

$$
\lim _{Q \rightarrow \infty} \frac{\Phi_{k}(Q ; B)}{Q^{n+1}}=\frac{2^{n}}{\zeta(n+1)} \int_{B} \rho_{k}(\mathbf{x}) d \mathbf{x}
$$

верно для любой фиксированной области $B$, у которой граница $\partial B$ имеет нулевую меру Лебега. Однако чтобы дать равномерную оценку скорости сходимости, нужно рассматривать области $B$ с более конкретными условиями на “извилистость” границы $\partial B$.

Наличие множителей вида $\left|x_{i}-x_{j}\right|$ означает, что мы имеем дело с детерминантным процессом, и корни как бы "расталкивают" друг друга.

Ясно, что при $k=1$ получается предыдущая теорема. При $k=n$ получается плотность распределения вполне вещественных алгебраических чисел:

$$
\rho_{n}(\mathbf{x})=\frac{2^{-n}}{n+1}\left(\frac{1}{\max _{0 \leqslant i \leqslant n}\left|\sigma_{i}(\mathbf{x})\right|}\right)^{n+1} \prod_{1 \leqslant i<j \leqslant n}\left|x_{i}-x_{j}\right| .
$$

Ясно, что $\rho_{k}(\mathbf{x})$ инвариантна относительно перестановок координат и умножения $\mathbf{x}$ на -1 . Чуть менее очевидно такое свойство:

$$
\rho_{k}\left(x_{1}^{-1}, \ldots, x_{k}^{-1}\right)=\rho_{k}\left(x_{1}, \ldots, x_{k}\right) \prod_{i=1}^{k} x_{i}^{2} .
$$

М. А. Королёв (Математический институт им. В. А. Стеклова Российской академии наук, г. Москва), Об оценках сумм Клоостермана методом А. А. Карацубы

Пусть $q \geqslant 3$ - натуральное число и $A \subset(\mathbb{Z} / q \mathbb{Z})^{*}$ - подмножество приведенной системы вычетов по модулю q. Сумма

$$
S_{q}(A ; a, b)=\sum_{n \in A} e_{q}\left(a n^{*}+b n\right)
$$

где $e_{q}(x)=e^{2 \pi i x / q}$ и $n n^{*} \equiv 1 \bmod q$, называется суммой Клоостермана. Если мощность множества $A$ очень мала по сравнению с $q$, например $|A|<q^{1-c}$, то сумма Клоостермана называется короткой.

Будем далее рассматривать в качестве множества $A$ отрезок $1 \leqslant n \leqslant N$. При $N \geqslant q^{1 / 2+\varepsilon}$ метод Вейля позволяет доказать, что

$$
S_{q}(A ; a, b) \ll \sqrt{q} \ln q .
$$

В 1990-е гг. А. А. Карацуба создал метод, дающий нетривиальные оценки сумм $S_{q}(A, a, b)$ уже в случае $N \asymp q^{\varepsilon}$. В основе метода лежит оценка количества $I_{k}(q ; N)$ решений сравнения

$$
x_{1}^{*}+\cdots+x_{k}^{*} \equiv x_{k+1}^{*}+\cdots+x_{2 k}^{*} \bmod q
$$

в целых числах $1 \leqslant x_{1}, \ldots, x_{2 k} \leqslant N$. 
А. А. Карацуба доказал близкую к оптимальной оценку

$$
I_{k}(q ; N) \leqslant(2 k)^{k^{3}} N^{k}(\ln N)^{k^{2}},
$$

которая нетривиальна при

$$
k \leqslant k_{0}(q) \asymp\left(\frac{\ln q}{\ln \ln q}\right)^{1 / 3} .
$$

Эвристически из этого следует, что нетривиальные оценки сумм Клоостермана должны получаться уже при

$$
N \geqslant e^{c_{1}(\ln q)^{2 / 3+\varepsilon}} \text {. }
$$

Первые результаты в этом направлении были несколько слабее: в середине 90-х гг. А. А. Карацуба доказал нетривиальную оценку с логарифмическим понижением в случае $N=q^{\varepsilon}$, позже подобная оценка была получена при $N \geqslant e^{c(\ln q)^{4 / 5+\varepsilon}}$.

В 2015 г. Ж. Бургейн и М. З. Гараев доказали, что при $N \geqslant e^{c(\ln q)^{2 / 3}(\ln \ln q)^{5}}$ имеет место оценка

$$
S_{q}(A ; a, 0) \ll N \frac{\ln q}{(\ln N)^{3 / 2}}(\ln \ln q)^{3} .
$$

Их доказательство опирается на весьма сложные и глубокие результаты из аддитивной комбинаторики. Докладчику удалось показать, что ряд оценок, подобных оценке Бургейна и Гараева, и даже чуть более точных, можно получить, заменив в аргументации этих авторов аддитивно-комбинаторные методы на элементарные (методом Карацубы и оригинальным соображением П. В. Снурницына). В частности, справедливы следующие утверждения.

ТЕорема 1. При простом $q$ и $N \geqslant e^{c(\ln q)^{2 / 3}(\ln \ln q)^{4 / 3}}$ имеет место оченка

$$
\sum_{0<x \leqslant N} e_{q}\left(a x^{*}\right) \ll N \frac{\ln q}{(\ln N)^{3 / 2}}(\ln \ln q)^{2} .
$$

Теорема 2. Пустъ q простое, $N \geqslant e^{c(\ln q)^{2 / 3}(\ln \ln q)^{1 / 3}}$. Тогда выполнено неравенство

$$
\sum_{0<x \leqslant N} e_{q}\left(a x^{*}\right) \ll \frac{N \ln \mathcal{D}}{\mathcal{D}}, \quad \text { əде } \quad \mathcal{D}=\frac{\ln N}{(\ln q)^{2 / 3}(\ln \ln q)^{1 / 3}} .
$$

Теорема 3. В условиях предыдущей теоремы справедлива оценка

$$
\sum_{0<x \leqslant N} e_{q}\left(a x^{*}+b x\right) \ll \frac{N}{\mathcal{D}^{3 / 4}} .
$$

Также удалось доказать, что для простого $q$ и целого $N$ с условиями $\ln N \asymp \ln q, N \leqslant q^{1 / 2}$, лежащего в промежутках специального вида, имеет место оценка

$$
S_{q}(A ; a, b) \ll \frac{N}{(\ln N)^{1-\varepsilon}} .
$$

Это усиливает оценку Бургейна и Гараева, понижение в которой для рассматриваемых $N$ имеет порядок $(\ln N)^{-1 / 2}(\ln \ln N)^{2}$.

Н. Г. Мощевитин (Московский государственный университет имени М.В. Ломоносова, механико-математический факультет), Новый взгляд на теорему Касселса о малых нулях квадратичных форм

Пусть

$$
Q(x)=\sum_{i, j \leqslant d} Q_{i j} x_{i} x_{j}
$$


где $Q_{i j}=Q_{j i} \in \mathbb{Z}$ - невырожденная квадратичная форма от $d$ переменных. Будем говорить, что форма $Q$ изотропна, если существует такое $g \in \mathbb{Z}^{d} \backslash\{0\}$, что $Q(g)=0$.

Теорема 1 (А. Мейер). Если невырожденная форма от пяти и более переменных представляет 0 в $\mathbb{R}$, то она изотропна.

Пусть теперь в предыдущих обозначениях $Q=\max _{i, j}\left|Q_{i j}\right|$. Тогда имеет место следующая оценка Касселса для нулей изотропных форм.

Теорема 2 (Дж.В.С. Касселс). Если $Q$ - изотропная квадратичная форма от $d$ nеременных, то существует $g \in \mathbb{Z}^{d} \backslash\{0\}$ с $Q(g)=0 u \max \left|g_{i}\right| \leqslant(3 Q)^{(d-1) / 2}$.

Позднее был получен ряд результатов, уточняющих теорему Касселса в различных направлениях.

ТЕОРема 3 (Г. П. Шликевай). Если $Q$ обращается в нуль на $\tau$-мерном рациональном подпространстве, то существует нетривиальный целочисленный нуль $g \in \mathbb{Z}^{d} c$

$$
\max \left|g_{i}\right| \ll Q^{(d-\tau) /(2 \tau)} .
$$

ТеоремА 4 (Р. Шульце-Пиллот). Если $Q$ - изотропная квадратичная форма, то существует набор из $d$ линейно независимых векторов $g^{\nu}=\left(g_{1}^{\nu}, \ldots, g_{d}^{\nu}\right), 1 \leqslant \nu \leqslant d, c Q\left(g^{\nu}\right)=0 u$

$$
\max _{k, \nu}\left|g_{k}^{\nu}\right| \ll Q^{(d-1) / 2} .
$$

Также эта тематика тесно связана с так называемыми внутренними рациональными приближениями.

Теорема 5 (Д. Я. Клейнбок, К. Меррил). Пустъ $S^{d-1} \subset \mathbb{R}^{d}-$ единичная $(d-1)$-мерная сфера. Тогда для любого $\alpha \in S^{d-1} \backslash \mathbb{Q}^{d}$ существует бесконечно много рачиональных точек

$$
\frac{p}{q}=\left(\frac{p_{1}}{q}, \ldots, \frac{p_{d}}{q}\right) \in S^{d-1} \cap \mathbb{Q}^{d}
$$

таких, что выполнено неравенство

$$
\max _{j}\left|\alpha_{j}-\frac{p_{j}}{q}\right| \leqslant \frac{C_{d}}{q}
$$

(здесъ $C_{d}-$ некоторая неэффективная константа, зависящая от $d$ ).

Эта теорема была обобщена ее авторами, а также Фишманом и Симмонсом для случая произвольных положительно определенных форм. Все эти теоремы могут рассматриваться как количественные варианты некоторой теоремы Касселса о приближениях.

Оказывается, для некоторых специальных форм выполняется более сильная оценка.

ТЕорема 6. Пусть $f(x)$ - квадратичная форма от $n$ переменных, и пусть $F(z)=$ $F(x, y)=f(x)-y^{2}$. Обозначим $\mathfrak{v}_{f}=\operatorname{vol}\{x|| f(x) \mid<1\}$. Если $F$ изотропна, то для всякого $\alpha$ с хотя бы одной иррачиональной координатой и $f(\alpha)=1$ существует бесконечно много maкux

$$
r=\left(\frac{a_{1}}{q}, \ldots, \frac{a_{n}}{q}\right)
$$

c $q, a_{i} \in \mathbb{Z}$, чmo $f(r)=1 u$

$$
\sqrt{f(\alpha-r)} \leqslant \frac{3 \max \left\{1,(n+1)^{n+1} 6^{n} 2^{n^{2}} / \mathfrak{v}_{f}^{n+1}\right\}}{q} .
$$


У. М. Пачев (Кабардино-Балкарский государственный университет им. Х. М. Бербекова, г. Нальчик), О применении дискретного эргодического метода в аналитической теории диобантовых уравнений

Будем рассматривать уравнение $x^{2}+y^{2}+z^{2}=m$ в целых числах при $m \rightarrow+\infty$. Количество решений $r(m)$ выражается через число классов квадратичных форм дискриминанта $(-m)$ следующим образом:

$$
r(m)= \begin{cases}12 h(-m), & \text { если } m \equiv 1,2(\bmod 4), \\ 8 h(-m), & \text { если } m \equiv 3(\bmod 8) .\end{cases}
$$

Подсчет количества решений уравнения $f\left(x_{1}, \ldots, x_{n}\right)=m$, где $f$ - целочисленная квадратичная форма, - одно из классических направлений в теории чисел. При $n \geqslant 4$ к задачам такого рода применим круговой метод, который, однако, оказывается бессилен при $n=3$. В связи с этим Ю. В. Линник разработал так называемый дискретный эргодический метод.

В основе метода лежит использование кватернионной арифметики. Пусть

$$
a+b i+c j+d k=Q \in \mathbb{H}
$$

- липшицев кватернион, $(a, b, c, d) \in \mathbb{Z}^{4}$. Будем называть величину $b i+c j+d k$ кватернионным вектором. Далее, если $l \in \mathbb{R}$, а $L-$ кватернионный вектор с условием $l+L=Q R$, то, переставляя множители, получим $l+L^{\prime}=R Q$.

Таким образом, арифметические свойства кватернионов позволяют по решению $(b, c, d)$ уравнения $b^{2}+c^{2}+d^{2}=m$ строить новое решение $\left(b^{\prime}, c^{\prime}, d^{\prime}\right)$. Продолжая этот процесс, можно получать цепочки кватернионных векторов.

При помощи своего метода Линнику удалось доказать ряд утверждений о равнораспределенности целых точек на сфере. Докладчик продемонстрировал, что эти же методы работают и для неопределенных форм.

Иными словами, справедливы асимптотические формулы для количеств решений уравнения $f\left(x_{1}, x_{2}, x_{3}\right)=m$, где $f$ - подходящая неопределенная квадратичная форма (например, $\left.x_{1} x_{3}-x_{2}^{2}\right)$, а $\left(x_{1}, x_{2}, x_{3}\right) \in \Lambda$, где $\Lambda$ - некоторая квадрируемая область на гиперболоиде $f\left(x_{1}, x_{2}, x_{3}\right)=m$.

Д. А. Попов (Московский государственный университет имени М. В. Ломоносова; Научно-исследовательский институт физико-химической биологии имени А.Н. Белозерского), Дискретный спектр оператора Лапласа на фундаментальной области модулярной группь и функиия Чебъшёва

Пусть $\Gamma=\mathrm{SL}(2, \mathbb{Z}), \mathcal{H}$ - верхняя полуплоскость с метрикой $d s^{2}=y^{-2}\left(d x^{2}+d y^{2}\right), \mathcal{F}=$ $\Gamma \backslash \mathcal{H}$ - фактор по действию $Г$. Можно рассмотреть дискретный спектр лапласиана на $\mathcal{F}$, т.е. множество всех таких $\lambda$, для которых существует функция $0 \neq \varphi \in L^{2}\left(\mathcal{F}, d x d y / y^{2}\right)$, удовлетворяющая уравнению

$$
\Delta \varphi+\lambda \varphi=0
$$

где

$$
\Delta=y^{2}\left(\frac{\partial^{2}}{\partial x^{2}}+\frac{\partial^{2}}{\partial y^{2}}\right) .
$$

Одна из причин, обуславливающих интерес к собственным функциям лапласиана на $\mathcal{F}$, состоит в том, что всякая такая функция продолжается до неголоморфной автоморфной формы, т.е. формы Маасса. Гипотеза Сельберга утверждает, что для всякой группы конечного кообъема дискретный спектр соответствующего лапласиана бесконечен и в некотором смысле достаточно полон. Гипотеза Сарнака-Филиппса говорит также, что для кофинитной группы, отвечающей точке общего положения в пространстве Тейхмюллера, дискретный спектр исчезает при деформациях комплексной структуры. 
Для $\mathrm{SL}(2, \mathbb{Z})$ и вообще для произвольной кофинитной группы можно рассматривать два спектра - спектр лапласиана, а также так называемый спектр резонансов. В случае $\operatorname{SL}(2, \mathbb{Z})$ спектр резонансов с точностью до некоторых тривиальных добавок, есть множество нетривиальных нулей дзета-функции Римана. В 1978 г. А. Б. Венков доказал следующую теорему, связывающую эти два спектра для $\mathrm{SL}(2, \mathbb{Z})$.

ТеОрема 1. Пусть при $m \in \mathbb{N}$ имеем $\lambda_{m}=1 / 4+r_{m}^{2}$, где $\left\{\lambda_{m}\right\}$ - дискретный спектр лапласиана на $\mathcal{F}$. Тогда при $t \rightarrow 0$ имеем

$$
\sum_{m=0}^{+\infty} e^{-t r_{m}^{2}} \cos \left(a r_{m}\right)= \begin{cases}\frac{1}{2 \sqrt{\pi t}} \frac{\Lambda(n)}{n}+O(1), & \text { если } a=2 \ln n, n \in \mathbb{N}, \\ \frac{1}{4 \sqrt{\pi t}} \frac{\ln N(P)}{\sqrt{N(P)}-1 / \sqrt{N(P)}}+O(1), & \text { если } a=\ln N(P), \\ O(1) & \text { в остальных случаях },\end{cases}
$$

где $N(P)$ - собственные значения гиперболических элементов в $\mathrm{SL}(2, \mathbb{Z})$.

Теорема Венкова неравномерна по $n$ (что проявляется особенно ярко в случае, когда $2 \ln n \approx \ln N(P)$ ). Таким образом, эта теорема не позволяет полностью восстановить функцию Мангольдта по дискретному спектру лапласиана на $\mathcal{F}$.

Докладчику удалось, опираясь на формулу следа Сельберга, вывести тождество, решающее эту проблему.

Теорема 2 (Попов). Пусть $x \geqslant 3 u 0<t \leqslant p^{-1} x^{-4}(\ln x)^{-1}$, где $p \geqslant 20-$ фиксированная постоянная. Тогда справедлива следующая формула для $\psi$-функиии Чебышёва:

$$
\psi(x)=2 \sqrt{\pi} t \sum_{m=0}^{+\infty} e^{-t r_{m}^{2}} \sum_{2 \leqslant k \leqslant x} k \cos \left(2 r_{m} \ln k\right)+R(x),
$$

¿əe

$$
|R(x)| \leqslant \frac{c x^{2} \sqrt{t}}{(\ln x)^{2}} \ll \frac{1}{(\ln x)^{5 / 2}} .
$$

Не исключено также, что по дискретному спектру можно полностью восстановить спектр резонансов, но этого сделать пока не удалось.

3. X. Рахмонов (Институт математики АН Республики Таджикистан, г. Душанбе), Суммы характеров по свободному от кубов модулю на сдвинутых простых

Пусть $q$ - простое число, $(l, q)=1, \chi$ - неглавный характер по модулю $q$. В 1938 г. И. М. Виноградов доказал, что для всякого $\varepsilon>0$ выполнено неравенство

$$
T(\chi ; x):=\sum_{p \leqslant x} \chi(p-l) \ll x^{1+\varepsilon}\left(\sqrt{\frac{1}{q}+\frac{q}{x}}+x^{-1 / 6}\right) .
$$

Эта оценка нетривиальна при $x \gg q^{1+\varepsilon}$. В 1952-1953 гг. Виноградов получил оценки, нетривиальные при $x \geqslant q^{3 / 4+\varepsilon}$. Этот результат не следует даже из гипотезы Римана.

А. А. Карацубой получены результаты, усиливающие оценки Виноградова и дающие нетривиальную оценку суммы $T(\chi ; x)$ уже при $x \geqslant q^{1 / 2+\varepsilon}$, а именно

Теорема 1 (А.А. Карацуба). Пусть $q$ - простое число, ұ-неглавный характер по модулю $q, x \geqslant q^{1 / 2+\varepsilon}$. Тогда имеет место оценка

$$
T(\chi, x) \ll x q^{-(1 / 1024) \varepsilon^{2}} .
$$


Также метод А. А. Карацубы позволяет улавливать и некоторую независимость различных сдвигов простых чисел по модулю $q$.

Теорема 2 (А. А. Карацуба). Пусть $\tau_{1}= \pm 1, \tau_{2}= \pm 1, q-$ простое число, $k_{1}$ u $k_{2}$ - целье числа такие, что $q \nmid k_{1} k_{2}\left(k_{1}-k_{2}\right), \omega \in(0,1 / 2), x \geqslant q^{0.75+\varepsilon}$. Пусть $T-$ количество простых $p \leqslant x$ с условиями

$$
\left(\frac{p+k_{1}}{q}\right)=\tau_{1}, \quad\left(\frac{p+k_{2}}{q}\right)=\tau_{2} .
$$

Тогда справедливо равенство

$$
T=\frac{1}{4} \pi(x)+O_{\omega}\left(x q^{-0.01 \omega^{2}}\right) .
$$

В 1985 г. докладчиком был доказан аналог оценки Виноградова для составных модулей, нетривиальный при $x \geqslant q \ln ^{13} q$.

Теорема 3 (З. Х. Рахмонов). Пусть D - достаточно большое положстельное число, $\chi-$ неглавный характер по модулю $D, \chi_{q}$ - примитивный характер по модулю q, индуиирующий $\chi$. Тогда выполняется неравенство

$$
T(\chi ; x) \leqslant x \ln ^{5} x\left(\sqrt{\frac{1}{q}+\frac{q}{x} \tau\left(q_{1}\right)^{2}}+x^{-1 / 6} \tau\left(q_{1}\right)\right),
$$

где $q_{1}=\prod_{\substack{p \mid D \\ p \nmid q}} p$. Ecлu $\chi=\chi_{q}$, mo

$$
T(\chi ; x) \leqslant x \ln ^{5} x\left(\sqrt{\frac{1}{q}+\frac{q}{x}}+x^{-1 / 6}\right) .
$$

В 2010 г. Дж. Б. Фридлендер, К. Гонг и И. Е. Шпарлинский доказали нетривиальную оценку для $T(\chi ; x)$ в случае, когда характер $\chi$ примитивный и $x \geqslant q^{8 / 9+\varepsilon}$. Через три года докладчик смог усилить этот результат.

Теорема 4 (З.Х. Рахмонов, 2013). Пусть q достаточно велико, $\chi_{q}$ - примитивный $x а-$ рактер по модулю $q, l$ взаимно просто с $q, \varepsilon>0, \mathcal{L}=\ln q, x \geqslant q^{5 / 6+\varepsilon}$. Тогда справедлива оценка

$$
T\left(\chi_{q} ; x\right)=\sum_{p \leqslant x} \chi_{q}(p-l) \ll x e^{-\sqrt{\mathcal{L}}} .
$$

Наконец, удается получить оценку, нетривиальную при тех же условиях, что и оценка из теоремы 1.

Теорема 5 (З.Х. Рахмонов). Пусть D - достаточно большое натуральное число, $\chi-$ неглавный характер по модулю $D, \chi_{q}$ - примитивный характер по модулю $q$, порожденныц характером $\chi, q$ - свободное от кубов, $(l, D)=1, \varepsilon \leqslant 10^{-3}$ - фиксированное положительное число. Тогда при $x \geqslant D^{1 / 2+\varepsilon}$, имеем

$$
T(\chi)=\sum_{p \leqslant x} \chi(p-l) \ll x e^{-0,6 \sqrt{\ln D}},
$$

где постоянная под знаком « зависит только от $\varepsilon$.

Доказательство этой теоремы комбинирует методы А.А. Карацубы и И. М. Виноградова с методом доказательства теоремы 4, а также опирается на оценки А. И. Виноградова и Д. А. Бёрджесса для сумм характеров. 
И. С. Резвякова (Математический институт им. В. А. Стеклова Российской академии наук, г. Москва), О методе Сельберга и аддитивной проблеме в задачах теории L-функиий

Будем говорить, что функция $L(s)$ лежит в классе Сельберга, если выполнены следующие пять аксиом:

1) $L(s)=\sum_{n=1}^{+\infty}\left(a(n) / n^{s}\right)$, где ряд в правой части сходится абсолютно при $\operatorname{Re} s>1$;

2) $(s-1)^{m} L(s)$ - целая функция конечного порядка для некоторого $m \geqslant 0$;

3) $a(1)=1$ и для всякого $\varepsilon>0$ выполнено неравенство $a(n) \ll_{\varepsilon} n^{\varepsilon}$;

4) $L(s)$ разлагается в эйлерово произведение по простым числам;

5) $L(s)$ удовлетворяет функциональному уравнению вида

$$
\Lambda(s):=H_{L}(s) L(s)=\overline{\Lambda(1-\bar{s})} .
$$

Гипотетически все $L$-функции из класса Сельберга удовлетворяют гипотезе Римана. Можно попробовать получать различные приближения к гипотезе Римана для данной $L$-функции $L(s)$. Оказывается, для этого нужно научиться решать аддитивные проблемы для коэффициентов $L(s)$, а именно получать нетривиальные оценки (либо выделять главный член и получать нетривиальные оценки остатка) для сумм вида

$$
S=\sum_{\substack{a n-b m=\ell \\ n \leqslant N}} a(n) \overline{a(m)}
$$

где $a, b, \ell$ - положительные целые числа, быть может, умеренно возрастающие вместе с ростом $N$.

Метод, разработанный А. Сельбергом, позволяет выводить из аддитивных задач с коэффициентами множество фактов, связанных с $L$-функциями и гипотезой Римана для них. В частности, получаются оценки для величин

$$
\int_{T}^{2 T}\left|L\left(\frac{1}{2}+i t\right)\right|^{2} d t, \quad \int_{T}^{2 T}\left|L\left(\frac{1}{2}+i t\right) \eta\left(\frac{1}{2}+i t\right)\right|^{2} d t
$$

где $\eta(s)$ - многочлен Дирихле с достаточно малым количеством слагаемых.

Кроме того, удается доказать аналог плотностной теоремы для $\zeta(s)$.

ТЕорема 1 (плотностная теорема). Пусть $N(\sigma, T)$ - количество нулей $L(s)$ в области $\operatorname{Re} s \geqslant \sigma,|\operatorname{Im} s| \leqslant T$. Тогда существует такая константа а $>0$, что при $1 \geqslant \sigma \geqslant 1 / 2$ выполнена оценка

$$
N(\sigma, T) \ll T^{1-a(\sigma-1 / 2)} \ln T .
$$

Из этой теоремы следует также теорема о распределении значений $L(s)$.

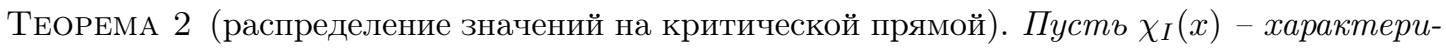
стическая функция отрезка $I \subset \mathbb{R}$. Тогда имеет место равенство

$$
\int_{T}^{2 T} \chi_{I}\left(\frac{\ln |L(1 / 2+i t)|}{\sqrt{c_{L} \pi \ln \ln T}}\right) d t=T \int_{I} e^{-\pi u^{2}} d u+o(T),
$$

причем слагаемое с о (T) при помощи аргументов А. Гоша и К.-М. Цанга можно заменить на

$$
O\left(\frac{T}{\sqrt{\ln \ln \ln T}}\right) \quad u \quad O\left(\frac{T(\ln \ln \ln T)^{2}}{\sqrt{\ln \ln T}}\right)
$$

соответственно. 
Метод, разработанный Сельбергом, опирается на правильный выбор так называемого "успокаивающего множителя", т.е. многочлена Дирихле $\eta(s)$, который понижает порядок величины

$$
\int_{T}^{2 T}\left|L\left(\frac{1}{2}+i t\right) \eta\left(\frac{1}{2}+i t\right)\right|^{2} d t
$$

Можно также рассматривать линейные комбинации $L$-функций из класса Сельберга, которые не имеют эйлерового произведения, однако удовлетворяют функциональному уравнению. Хорошо известно, что для таких функций гипотеза Римана неверна. Тем не менее оказывается, что аномально много нулей лежат на критической прямой. Более того, методом Сельберга можно доказать, что положительная доля нулей такой линейной комбинации лежит на критической прямой.

Результаты такого рода для линейных комбинаций $L$-функций основаны на следующем аналоге теоремы 2.

ТеОРема 3. Пусть $L_{1}(s), L_{2}(s)$ - независимые L-функиии из класса Селъберга. Тогда для любого отрезка $I$ выполнено равенство

$$
\int_{T}^{2 T} \chi_{I}\left(\frac{\ln \left|L_{1}(1 / 2+i t)\right|-\ln \left|L_{2}(1 / 2+i t)\right|}{\sqrt{\left(n_{1}+n_{2}\right) \pi \ln \ln T}}\right) d t=T \int_{I} e^{-\pi u^{2}} d u+O\left(\frac{T(\ln \ln \ln T)^{2}}{\sqrt{\ln \ln T}}\right) .
$$

Вывод из этой теоремы факта о положительной доле нулей использует такую идею: согласно теореме 3, за исключением множества небольшой меры, значения одной из наших $L$-функций (примерно на половине отрезка - первой и на половине - второй) существенно больше значений другой, и потому их линейная комбинация ведет себя почти как доминирующая $L$-функция. Такое рассуждение упрощает выбор успокаивающего множителя и в итоге приводит к доказательству.

М. Ризагер (Копенгагенский университет), Гиперболическая проблема круга

Классическую проблему круга Гаусса (или евклидову проблему круга) можно сформулировать следующим образом: для положительного $R$ определим

$$
N_{E}(R)=\#\left\{\gamma \in \mathbb{Z}^{2} \mid d_{E}(\gamma(0), 0) \leqslant R\right\}
$$

где $d_{E}(x, y)$ - евклидово расстояние между точками $x$ и $y$, а $\gamma$ рассматривается как элемент $\operatorname{Isom}\left(\mathbb{R}^{2}\right)$. Тогда $N_{E}(R)=\pi R^{2}+E(R)$ и $E(R)=O(R)$. При помощи формулы суммирования Пуассона можно получить такую формулу для $E(R)$ :

$$
E(R)=R^{1 / 2} \sum_{m>0} \frac{r_{2}(m)}{m^{3 / 4}} \cos \left(2 \pi R \sqrt{m}-\frac{3 \pi}{4}\right) \psi\left(\frac{m}{T}\right)+O\left(R^{1 / 2}+\frac{R^{1+\varepsilon}}{T^{1 / 2}}\right),
$$

где $\psi(x)$ - правильная сглаживающая куполообразная функция.

Если в последем равенстве сумму по $m$ оценить тривиально, то получится оценка В. Ф. Серпинского $E(R) \ll R^{2 / 3+\varepsilon}$. Наилучший на данный момент результат - оценка М. Хаксли вида $E(R) \ll R^{131 / 208+\varepsilon}$, в то время как гипотеза $\Gamma$.Х. Харди предсказывает $E(R) \ll R^{1 / 2+\varepsilon}$.

Можно рассматривать аналог проблемы круга для гиперболической плоскости: пусть $\Gamma$ фуксова группа, действующая на верхней полуплоскости $\mathcal{H}$ и имеющая конечный кообъем. По аналогии с $N_{E}(R)$ определим

$$
N_{H}(R, z)=\#\left\{\gamma \in \Gamma \mid d_{H}(\gamma z, z) \leqslant R\right\} .
$$

Можно предположить, что главный член здесь будет равен

$$
\frac{\operatorname{vol}(B(z, R))}{\operatorname{vol}(\Gamma \backslash \mathcal{H})}=: M(R)
$$


Пусть $E(R, z)=N_{H}(R, z)-M(R)$ - остаточный член. Аналогом формулы суммирования Пуассона в этом случае является формула следа Сельберга. Она дает следующее выражение для остатка:

$$
E(R, z)=\sqrt{\pi} e^{R / 2} \sum_{t_{j}>0} \operatorname{Re}\left(e^{i R t_{j}} \frac{\Gamma\left(i t_{j}\right)\left|\varphi_{j}(z)\right|^{2}}{\Gamma\left(3 / 2+i t_{j}\right)}\right) \psi\left(\frac{t_{j}}{T}\right)+O\left(\frac{e^{R}}{T}+e^{R(1 / 2+\varepsilon)}\right)
$$

где $t_{j}$ и $\varphi_{j}(z)$ - собственные значения и соответствующие собственные функции автоморфного лапласиана $-\Delta$. Оценивая тривиально, можно доказать оценку $E(R, z) \ll e^{R(2 / 3+\varepsilon)}$. Это результат Сельберга и наилучшая из доказанных на данный момент оценок. Докладчику удалось доказать две теоремы, усиливающие эту оценку в среднем. Первая из них использует аналитическое усреднение.

Теорема 1 (Я.Н. Петридис, М. Ризагер, 2014). Пусть $\Gamma=S L_{2}(\mathbb{Z})$ u $f$ - гладкая неотрицательная функция на $Г \backslash \mathcal{H}$ с компактным носителем. Тогда выполнена оченка

$$
\int_{\Gamma \backslash \mathcal{H}} f(z)(N(R, z)-M(R)) d \mu(z)=O\left(e^{R(7 / 12+\varepsilon)}\right) .
$$

Можно также усреднять величину $N(R, z)-M(R)$ арифметически. А именно, пусть $-D<0$ - фундаментальный дискриминант и $\mathfrak{F}_{D}-$ множество квадратичных форм такого дискриминанта. Группа $S L_{2}(\mathbb{Z})$ действует на $\mathfrak{F}_{D}$. Пусть $p(x, y)=a x^{2}+b x y+c y^{2} \in \mathfrak{F}_{D}$, тогда

$$
\tau_{P}=\frac{-b+i \sqrt{D}}{2 a} \in \mathcal{H}
$$

называется точкой Хегнера дискриминанта -D. Обозначим все такие точки, лежащие внутри фундаментальной области $S L_{2}(\mathbb{Z})$, через $\Lambda_{D}$. Тогда $\left|\Lambda_{D}\right|=h(-D)$. При арифметическом усреднении выполнен такой факт.

Теорема 2 (Я.Н. Петридис, М. Ризагер). Пусть $Г=S L_{2}(\mathbb{Z})$ u $f$ - гладкая неотрицательная функиия на $Г \backslash \mathcal{H}$ с компактным носителем. Тогда

$$
\frac{1}{h(-D)} \sum_{z \in \Lambda_{D}} f(z)(N(R, z)-M(R))=O\left(e^{R(7 / 12+\varepsilon)}+e^{R(4 / 5+\varepsilon)} D^{-4 / 165+\varepsilon}\right) .
$$

Эти оценки получаются с помощью теорем В. Луо, П. Сарнака об эффективной квантовой строгой эргодичности в среднем, Х. Иванца, В. Луо, П. Сарнака о сокращениях спектральных сумм, а также опираются на тождества типа Вальдшпурже и субвыпуклость типа Вейля в среднем. Кроме того, важна для доказательства следующая теорема.

Теорема 3 (Я.Н. Петридис, М. Ризагер; sup-нормы в среднем). Для всякого $\varepsilon>0 u$ всякого компакта $K$ выполнена оценка

$$
\sum_{T \leqslant t_{j} \leqslant 2 T} \sup _{z \in K}\left|\varphi_{j}(z)\right|^{2}=O_{K}\left((1+T)^{11 / 4+\varepsilon}\right) .
$$

А. А. Седунова (Университет Париж-юг 11), Обобщение метода Бомбъери-Пила на случай поля функиий

В 1989 г. Э. Бомбьери и Дж. Пила доказали, что если Г - подмножество неприводимой алгебраической кривой степени $d$, лежащее внутри квадрата со стороной $N$, то количество точек решетки, лежащих на $\Gamma$, не превосходит $c(d, \varepsilon) N^{1 / d+\varepsilon}$.

$\mathrm{У}$ этого результата имеется множество аналогов: например, можно было бы считать количество решений уравнения $f(x, y)=0$ в $\mathbb{F}_{p}$, попадающих в очень короткие (чтобы граница 
Вейля перестала работать) промежутки. Если продолжать эту аналогию, то наиболее естественно было бы попробовать доказать такое утверждение в функциональном случае, т.е. для кольца $\mathbb{F}_{q}[T]$. В этой ситуации аналогом короткого интервала будет выступать множество $X(T)+Y(T)$, где многочлен $X(T)$ фиксирован, а $Y(T)$ пробегает все многочлены степени $\leqslant n$.

Докладчиком было доказано следующее утверждение.

ТеОрема 1. Пусть $\mathcal{C}$ - неприводимая алгебраическая кривая степени $d$ над $\mathbb{F}_{q}[T]$. Если $S=\mathcal{C} \cap I^{2}$, где $I$ - множество всех полиномов в $\mathbb{F}_{q}[T]$ степени не выше $n$, то

$$
|S| \ll|I|^{1 / d+o(1)} .
$$

Выстраивать доказательство в полной аналогии с доказательством Э. Бомбьери и Дж. Пила не получается: в $\mathbb{F}_{q}[T]$ нет нужного аналога теоремы о среднем значении.

Пусть теперь $\mathcal{W}$ - конечный набор линейно независимых многочленов в $\left(\mathbb{F}_{q}[T]\right)[X, Y]$, такой, что $1 \in \mathcal{W}$. Будем обозначать $d_{\mathcal{W}}$ сумму степеней всех элементов $\mathcal{W}$ и $\omega=|\mathcal{W}|$. Будем также считать, что $\mathcal{W}$ разделяет точки. $\mathcal{W}$-Кривой называется аффинная алебраическая кривая $G(X, Y)=0$ такая, что все мономы $G$ принадлежат $\mathcal{W}$.

Методами в духе работы Х. Хельфготта, А. Венкатеша о плохо распределенных множествах докладчику удалось доказать следующую лемму.

ЛЕмма 1. Пусть $\mathcal{C}$ - неприводимая алгебраическая кривая степени d над $\mathbb{F}_{q}[T]$, a $S=$ $\mathcal{C} \cap I^{2}$. Предположим, что для некоторого фиксированного $\alpha>0$ и любого неприводимого полинома $f \in \mathbb{F}_{q}[T]$ количество вычетов $\{(X, Y) \bmod f \mid X, Y \in S\}$ не превосходит $\alpha q^{\operatorname{deg} f}$. Пусть множество $\mathcal{W}$ выбрано таким образом, что всякая $\mathcal{W}$-кривая содержит не более $C$ точек $S$. Тогда при больших $|I|$ имеет место соотношение

$$
|S| \ll \mathcal{W}|I|^{\frac{2 \alpha d_{\mathcal{W}}}{\omega(\omega-1)}+\psi(\omega, C, \alpha)},
$$

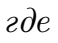

$$
\psi(\omega, C, \alpha)=\frac{2 \alpha d_{\mathcal{W}} C}{\omega^{2}(\omega-1)^{2}} .
$$

Применение леммы 1 для случая $\mathcal{W}=\mathcal{W}_{d-1, M}=\left\{X^{i} Y^{j} \mid i \leqslant d-1, j \leqslant M\right\}$ с достаточно большим $M$ позволяет, используя некоторые дополнительные аргументы с границей Вейля и выбором правильного многочлена для нашей кривой, доказать теорему 1. Выбор множества такой же, как в доказательстве Бомбьери и Пила, параметры из леммы равны

$$
\omega=d(M+1), \quad d_{\mathcal{W}}=d(M+1) \frac{d+M-1}{2} .
$$

Д. А. Фроленков (Математический институт им. В. А. Стеклова Российской академии наук, г. Москва), Усиление результата Портера

Пусть $q$ - рациональное число. Обозначим через $s(q)$ длину цепной дроби этого числа. Имеет место следующая асимптотическая формула для средней длины цепной дроби дробей с фиксированным знаменателем.

Теорема 1 (Дж. В. Портер, 1975). Для произвольного положительного є выполнено равенство

$$
\frac{1}{\varphi(b)} \sum_{\substack{1 \leqslant a \leqslant b \\(a, b)=1}} s\left(\frac{a}{b}\right)=\frac{2 \ln 2}{\zeta(2)} \ln b+C_{P}-1+O\left(b^{-1 / 6+\varepsilon}\right),
$$

әде

$$
C_{P}=\frac{2 \ln 2}{\zeta(2)}\left(\frac{3 \ln 2}{2}+2 \gamma-2 \frac{\zeta^{\prime}}{\zeta}(2)-1\right)-\frac{1}{2}
$$

- константа Портера. 
Оказывается, что оценка этой суммы связана с количеством решений одного уравнения в целых числах, а именно

Лемма 1 (Г. А. Хейльбронн, 1968; А. В. Устинов, 2005). Для всех $\varepsilon>0$ справедливо равенcm8o

$$
\sum_{\substack{1 \leqslant a \leqslant b \\(a, b)=1}} s\left(\frac{a}{b}\right)=2 \sum_{d_{1}, d_{2} \mid b} \mu\left(d_{1}\right) \mu\left(d_{2}\right) R\left(\frac{b}{d_{1} d_{2}}\right)-\frac{1}{2} \varphi(b)+O\left(b^{\varepsilon}\right),
$$

где $R(N)$ - количество решений уравнения $n_{1} n_{2}+n_{3} n_{4}=N$ с условиями $1 \leqslant n_{1} \leqslant n_{3} u$ $1 \leqslant n_{2} \leqslant n_{4}$.

Далее, при помощи сглаживающих функций и преобразования Меллина удается свести задачу улучшения остаточного члена в теореме 1 к вопросу об оценках суммы

$$
\sum_{n+m=N} \sigma_{s}(n) \sigma_{s}(m)
$$

где $\sigma_{s}(n)=\sum_{d \mid n} d^{s}$, т.е. к аддитивной проблеме делителей. Для получения таких оценок используется некоторый вариант формулы Вороного, что и приводит в итоге к следующему утверждению.

Теорема 2 (В.А. Быковский, Д. А. Фроленков). Для всякого $\varepsilon>0$ верна оценка

$$
\frac{1}{\varphi(b)} \sum_{\substack{1 \leqslant a \leqslant b \\(a, b)=1}} s\left(\frac{a}{b}\right)=\frac{2 \ln 2}{\zeta(2)} \ln b+C_{P}-1+O\left(b^{-6 / 29+\varepsilon}\right) .
$$

Г. В. Федоров (Московский государственный университет имени М. В. Ломоносова, механико-математический факультет; Научно-исследовательский институт системных исследований РАН, г. Москва), Теорема Римана-Роха и периодичность непрерывных дробей в гиперэллиптических полях

Одна из фундаментальных проблем теории чисел и алгебраической геометрии - описание кручения на якобианах гиперэллиптических кривых над $\mathbb{Q}$. Корни этой проблемы лежат еще в исследованиях XIX века.

Как известно, якобиан эллиптической кривой изоморфен самой кривой. Случай рода 1 решен над $\mathbb{Q}$ Б. Мазуром в 1978 г. В 2010 г. В. П. Платонову удалось осуществить продвижение в этой проблеме, основываясь на естественной связи между фундаментальными единицами (или, более широко, $S$-единицами) и точками кручения на якобианах гиперэллиптических кривых.

Связь между существованием нетривиальных $S$-единиц и периодичностью разложения в непрерывную дробь некоторых специальных элементов поля функций гиперэллиптической кривой в случае конечных нормирований была впервые обнаружена в совместной работе В. П. Платонова и докладчика (декабрь 2015 г.).

Их результаты позволяют получить быстрый алгоритм для построения фундаментальных $S$-единиц, используя подходящие дроби непрерывных дробей специальных элементов поля функций.

Пусть $K$ - некоторое поле (поле констант), char $K \neq 2, f \in K[x]$ - свободный от квадратов многочлен, $h(x) \in K[x], \operatorname{deg} h=1$. Конечное нормирование $\nu_{h}$ поля $K(x)$ определяется следующим образом: если $P, Q \in K[x]$ - не делящиеся на $h$ многочлены, а $m \in \mathbb{Z}$, то

$$
\nu_{h}\left(h^{m} \frac{P}{Q}\right)=m \text {. }
$$


Бесконечное нормирование $\nu_{\infty}$ определяется равенством

$$
\nu_{\infty}\left(\frac{P}{Q}\right)=\operatorname{deg} Q-\operatorname{deg} P .
$$

Связь между решениями уравнения Пелля $\omega_{1}^{2}-f \omega_{2}^{2}=1$ с $\omega_{i} \in \mathbb{C}[x]$ и периодическими непрерывными дробями в поле $\mathbb{C}((1 / x))$ была обнаружена еще в работах Н. Х. Абеля и П. Л. Чебышёва, посвященных гиперэллиптическим интегралам, т.е. интегралам вида

$$
\int \frac{P(x)}{\sqrt{f(x)}} d x .
$$

Пусть $f \in K[x]$ - многочлен нечетной степени $2 g+1, L=K(x)(\sqrt{f})$ - кольцо функций кривой $C: y^{2}=f(x)$. Тогда бесконечное нормирование имеет единственное продолжение с $K(x)$ на $L$. Пусть $\nu_{h}$ имеет два продолжения: $\nu_{h}^{-}$и $\nu_{h}^{+}$. Тогда всякий элемент $L$ раскладывается в непрерывную дробь в $K((h))$. Пусть теперь $S=\left\{\nu_{h}^{-}, \nu_{\infty}\right\}$, а $\mathcal{O}_{S}$ - кольцо $S$-целых элементов $L$. Мультипликативная группа $\mathcal{O}_{S}^{*}$ называется группой $S$-единиц.

Для конечного поля констант любая квадратичная иррациональность $\alpha$ в $L$ раскладывается в периодическую непрерывную дробь, поэтому фундаментальные $S$-единицы можно вычислять при помощи непрерывных дробей (это результат В. П. Платонова и В. В. Беняш-Кривеца).

В случае произвольного поля $K$ существование фундаментальной $S$-единицы оказывается эквивалентно периодичности разложения $\sqrt{f} h^{-g}$ или $\sqrt{f} h^{-g-1}$, и фундаментальные единицы можно вычислять при помощи подходящих дробей.

Напомним, как строятся непрерывные дроби. Пусть

$$
\alpha \in K((h)), \quad \alpha=\sum_{j=-r}^{+\infty} b_{j} h^{j},
$$

где $b_{j} \in K$. Определим $h$-целую часть как

$$
[\alpha]=[\alpha]_{h}=\sum_{j=-r}^{0} b_{j} h^{j}
$$

(если нижний предел больше верхнего, то сумму считаем нулевой). А имея функцию целой части, можно уже стандартным образом определить неполные частные и подходящие дроби непрерывной дроби.

Всякий элемент $\alpha \in L$ является корнем некоторого многочлена $H(X)=\lambda_{2} X^{2}+2 \lambda_{1} X+\lambda_{0}$, где $\lambda_{i} \in K[x]$ с условием $\left(\lambda_{0}, \lambda_{1}, \lambda_{2}\right)=1$. Для $j \in \mathbb{N}$ определим

$$
\begin{aligned}
& A_{j}=(-1)^{j+1}\left(\lambda_{2} p_{j}^{2}+2 \lambda_{1} p_{j} q_{j}+\lambda_{0} q_{j}^{2}\right), \\
& B_{j}=(-1)^{j}\left(\lambda_{2} p_{j} p_{j-1}+2 \lambda_{1} p_{j-1} q_{j}+\lambda_{0} q_{j} q_{j-1}\right),
\end{aligned}
$$

где $p_{j}$ и $q_{j}$ - числитель и знаменатель $j$-й подходящей дроби соответственно.

При помощи дивизоров на $C_{L}$ и формулы Римана-Роха докладчиком была доказана такая теорема.

ТЕОРЕма 1. Пусть свободный член многочлена $f=f(h)$ ненулевой и является квадратом в $K$. Пусть $s=g$ или $g+1, a \alpha=\sqrt{f} h^{-s}, a_{0}=[\alpha]$. Для некоторых $n, m \in \mathbb{N}$ следующие условия эквивалентны:

1) $n \geqslant 2$ - такое наименъшее $n$, что $\nu_{h}\left(A_{n-1}\right)=\operatorname{deg} A_{n-1}$;

2) непрерывная дробъ для $\alpha+a_{0} \in K((h))$ чисто периодична с периодом $n$ или $2 n$; 
3) $m$ - наименьшее натуральное число, для которого уравнение $\omega_{1}^{2}-f \omega_{2}^{2}=b h^{m}$ имеет решение с $\omega_{i} \in K[x], \nu_{h}\left(\omega_{1}\right)=0, b \in K^{*} ;$ в этом случае

$$
h^{-s} \frac{\omega_{1}}{\omega_{2}}=\frac{p_{n-1}}{q_{n-1}}
$$

- подходящая дробъ для $\alpha$

4) поле $L=K(x)(\sqrt{f})$ имеет фундаментальную $S$-единичу степени $m$;

5) класс дивизора $D=\nu_{h}^{-}-\nu_{\infty}$ имеет конечный порядок $m$ в группе $\Delta^{0}(L)$ классов дивизоров степени 0 поля $L$.

M. Е. Чанга (Московский педагогический государственный университет), О числах, количество простых делителей которых принадлежит заданному классу вычетов

Пусть $E$ - бесконечное подмножество в $\mathbb{N}, M-$ множество всех таких натуральных чисел $n$, у которых все простые делители принадлежат множеству $E$.

Пусть также $\gamma(n)$ - некоторая аддитивная функция такая, что $\gamma(p)=1$ для любого простого числа $p$. Наиболее классический выбор для $\gamma(n)$ - число простых делителей $n$ с учетом кратности $\Omega(n)$ и число различных простых делителей $\nu(n)$. Пусть $n(x)$ - считающая функция множества $M$. Также для любых целых $k \geqslant 2$ и $l$

$$
n_{l}(x ; k)=\sum_{\substack{n \leqslant x, n \in M \\ \gamma(n) \equiv l \bmod k}} 1 .
$$

Будем считать, что для множества $E$ выполнено хотя бы одно из двух условий: либо существуют такие положительные $c_{E}$ и $\Delta$, что

$$
\sum_{\substack{p \leqslant x \\ p \in E}} 1=c_{E} \pi(x)+O\left(x^{1-\Delta}\right)
$$

либо $E$ представимо в виде объединения $r$ различных (и не включающих одна другую) арифметических прогрессий по модулю $m$. В этом случае положим

$$
c_{E}=\frac{r}{\varphi(m)} .
$$

Один из примеров подходящего множества $E$, не являющегося объединением арифметических прогрессий, строится так: выберем натуральные $D$ и $j$, а также вещественное $a>0, a \notin \mathbb{N}$, и положим

$$
E=\mathbb{N} \cap \bigcup_{n=0}^{+\infty}\left[(D n+j-1)^{1 / a},(D n+j)^{1 / a}\right]
$$

В этом случае

$$
c_{E}=\frac{1}{D}
$$

Для всех “хороших" (т.е. имеющих перечисленные выше свойства) множеств $E$ имеет место асимптотика

$$
n(x) \sim \frac{C x}{(\ln x)^{1-c_{E}}} .
$$

Намного интереснее то, что происходит с $n_{l}(x ; k)$ при $k=2$. Оказывается, имеет место следующий факт. 
Теорема 1. Если $E$ “хорошее”, $E \neq \mathbb{N}$, то для некоторого положительного $A$ выполнено равенство

$$
n_{l}(x ; 2)=\frac{1}{2} n(x)\left(1+\frac{(-1)^{l+1} A}{\ln ^{2 c_{E}} x}+O\left(\ln ^{-2 c_{E}-1} x\right)\right) .
$$

Таким образом, при больших $x$ разность $n_{1}(x ; 2)-n_{0}(x ; 2)$ не только все время остается положительной, но и отличается по порядку от $n(x)$ всего лишь логарифмическим множителем. Это несколько неожиданно, поскольку при $E=\mathbb{N}$ можно показать, что

$$
n_{1}(x ; 2)-n_{0}(x ; 2) \ll n(x) e^{-c \sqrt{\ln x}} .
$$

Этот эффект был обнаружен А. А. Карацубой в случае $\gamma(n)=\Omega(n)$.

Посмотрим теперь на случай $k \geqslant 3$. Правда ли, что нам удастся найти такое $l \bmod k$, что $n_{l}(x ; k)$ доминирует над всеми остальными $n_{l^{\prime}}(x ; k)$ с $l^{\prime} \not \equiv l(\bmod k)$ при достаточно больших $x ?$

Оказывается, такого $l$ не существует ни для каких $k>2$ и ни для каких $E$, а именно, возникает некоторая осцилляция.

Теорема 2. Для всех $k \geqslant 3$ существуют такие константы $A, \varphi_{0} u \delta_{k}>2 c_{E} \sin ^{2}(\pi / k)$, чmo

$$
n_{l}(x ; k)=\frac{1}{k} n(x)\left(1+\frac{A \cos \left(c_{E} \sin (2 \pi / k) \ln \ln x+\varphi_{0}-2 \pi l / k\right)}{\ln ^{2 c_{E} \sin ^{2}(\pi / k)} x}+O\left(\ln ^{-\delta_{k}} x\right)\right) .
$$

Такая осцилляция и отсутствие “доминирующего" остатка обусловлены тем, что при применении метода комплексного интегрирования к этой задаче при $k>2$ приходится интегрировать $\zeta(s)$ в комплексной степени (с ненулевой мнимой частью).

В. Г. Чирский (Московский государственный университет имени М. В. Ломоносова), O представлениях натурального числа слагаемыми определенного вида

Пусть $P$ - некоторое конечное множество $\left\{p_{1}, p_{2}, \ldots, p_{t}\right\}$ простых чисел. Будем обозначать через $A(P)$ множество всех натуральных чисел, все простые делители которых лежат в $P$, и через $A_{ \pm}(P)$ - множество всех целых чисел с тем же свойством. Легко понять, что для всякого натурального $k$ существуют натуральные числа, не представимые никаким количеством $l \leqslant k$ слагаемых из $A(P)$ или $A_{ \pm}(P)$.

Можно также доказать, что для всякого натурального $k$ существует бесконечно много таких $n$, что $k$ - наименьшее количество слагаемых, которым $n$ может быть представлено в виде суммы чисел из $A_{ \pm}(P)$.

Пусть $F(k)$ и $F_{ \pm}(k)$ - наименьшие числа, не представимые суммой $k$ или меньше слагаемых из $A(P)$ и $A_{ \pm}(P)$ соответственно.

Тогда имеют место следующие оценки.

Теорема 1 (Л. Хайду, Р. Тайдеман, 2012). Для всякого конечного $P$ c $|P|=t$ и всякого $\varepsilon>0$ существуют константы с (зависит только от двух наименьших элементов в $P$ ), $C$ (зависит только от $\varepsilon$ ) и абсолютная константа $C_{ \pm}$такие, что:

1) $F(k)>k^{c k}$ npu $k>1$;

2) $F(k) \leqslant C(k t)^{(1+\varepsilon) k t}$ для всех $k>1$;

3) $F_{ \pm}(k)<e^{(k t)^{C_{ \pm}}}$npu $k>1$.

При $P=\{2,3\}$ в первой оценке получается $c \approx 0.000001$. Докладчику удалось существенно улучшить эту оценку при помощи рассуждения с «жадным» алгоритмом и одной леммы, доказанной В. Х. Салиховым.

Tеорема 2. Пусть $A=\left\{2^{a} 3^{b} \mid a, b \geqslant 0\right\}, A_{ \pm}=A \cup(-A)$. Пусть $F(k) u F_{ \pm}(k)-$ Hauменъшие натуральные числа, не представимые суммой $k$ или менъше слагаемых из $A$ u $A_{ \pm}$ 
соответственно. Тогда для всякого $\varepsilon>0$ существует $k_{0}$ такое, что при $k>k_{0}$ выполнены неравенства

$$
\left(\frac{k}{6}\right)^{k / 6} \leqslant F(k) \leqslant F_{ \pm}(k) \leqslant(2 k)^{(2+\varepsilon) k} .
$$

Из этой теоремы следует, что всякое достаточно большое натуральное число $n$ может быть представлено суммой чисел из $A$, содержащей не более $6 \ln n / \ln \ln n$ слагаемых.

Ключевую роль в доказательстве теоремы 2 играют одна лемма, доказанная В. Х. Салиховым и следующая знаменитая лемма Эвертса.

Лемма (Я.-Х. Эвертс, 2007). Пусть $c>0,0 \leqslant d<1, S_{0}=\{2,3\}$, a $l$ - натуралъное число. Тогда существует лишь конечное число наборов $\left(x_{0}, \ldots, x_{l}\right)$ целых чисел с условием НОД $\left(x_{0}, \ldots, x_{l}\right)=1$ таких, что $x_{0}+\cdots+x_{l}=0$, причем для всякого собственного подмножества $\left\{i_{1}, \ldots, i_{s}\right\} \subset\{0, \ldots, l\}$ выполнено $x_{i_{1}}+\cdots+x_{i_{s}} \neq 0, u$, кроме того,

$$
\prod_{j=0}^{l}\left(\left|x_{j}\right|\left|x_{j}\right|_{2}\left|x_{j}\right|_{3}\right) \leqslant c\left(\max \left|x_{j}\right|\right)^{d},
$$

где $|\cdot|_{p}-p$-адическая норма.

В. Н. Чубариков (Московский государственный университет имени М. В. Ломоносова, механико-математический факультет), Рациональные тригонометрические суммы и интегральь

Функция $\rho(x)=1 / 2-\{x\}$ играет важную роль во многих классических вопросах теории чисел, как то проблема делителей Дирихле, проблема круга Гаусса, формулы для числа классов квадратичных форм, квадратурные формулы и даже гипотеза Римана.

Зададимся целью научиться оценивать интегралы и суммы с участием $\rho(x)$. Одно из самых существенных для применений свойств нашей функции - это так называемая формула умножения, аналогичная формуле умножения для гамма-функции

$$
\rho(n x)=\sum_{k=0}^{n-1} \rho\left(x+\frac{k}{n}\right) .
$$

Аналогичным уравнениям удовлетворяют многочлены Бернулли, задающиеся с помощью равенства

$$
\frac{t e^{t x}}{e^{t}-1}=\sum_{n=0}^{+\infty} \frac{B_{n}(x) t^{n}}{n !} .
$$

Для интегралов, содержащих функцию $\rho(x)$, имеется серия оценок, аналогичных оценкам тригонометрических интегралов.

Теорема 1. Пусть для некоторого $A>0$ при всех $n>1$ u $x \in[0,1]$ выполнено неравество $\left|f^{(n)}(x)\right| \geqslant A$. Тогда справедлива верхняя ощенка

$$
|I|=\left|\int_{0}^{1} \rho(f(x)) d x\right| \leqslant \min \left\{1,4 n A^{-1 / n}\right\} .
$$

Эта оценка близка к оптимальной, так как в случае $f(x)=\alpha x^{n}$ можно показать, что

$$
|I| \geqslant \frac{1}{6} \alpha^{-1 / 6} .
$$


Tеорема 2. Пусть $n \geqslant 1, \alpha_{0}, \alpha_{1}, \ldots, \alpha_{n} \in \mathbb{R}, f(x)=\alpha_{n} x^{n}+\cdots+\alpha_{1} x+\alpha_{0}, \beta_{r}(x)=$ $s f^{(r)}(x) / r !, a$

$$
H=H(\bar{\alpha})=\min _{a \leqslant x \leqslant b} \max _{1 \leqslant r \leqslant n}\left|\beta_{r}(x)\right|^{1 / r} .
$$

Тогда справедлива оценка

$$
|J|=\left|\int_{a}^{b} \rho(f(x)) d x\right| \leqslant \min \left(b-a ; 4 e n^{2} H^{-1}\right) .
$$

Из этой теоремы можно вывести точную оценку показателя сходимости особого интеграла в проблеме Терри, в том числе для так называемых "выщербленных" многочленов.

Кроме того, имеется следующая оценка для интеграла с многочленом от большого числа переменных

TEOPEMA 3. Пусms

$$
F\left(x_{1}, \ldots, x_{n}\right)=\sum_{0 \leqslant t_{1}, \ldots, t_{r} \leqslant n} \alpha\left(t_{1}, \ldots, t_{r}\right) x_{1}^{t_{1}} x_{2}^{t_{2}} \ldots x_{r}^{t_{r}}, \quad \alpha=\max _{t_{1}+\cdots+t_{r} \geqslant 1}\left|\alpha\left(t_{1}, \ldots, t_{r}\right)\right| .
$$

Тогда имеет место следующая верхняя оценка:

$$
\left|I_{r}\right|=\left|\int_{[0,1]^{r}} \rho\left(F\left(x_{1}, \ldots, x_{r}\right)\right) d x_{1} \ldots d x_{r}\right| \leqslant \min \left(1,(8 e)^{r} \alpha^{-1 / n} \ln ^{r-1}(\alpha+2)\right) .
$$

Перейдем теперь к суммам, содержащим функцию $\rho(x)$.

Пусть $f(x)=a_{n} x^{n}+\cdots+a_{1} x+a_{0}$. Обозначим

$$
S=S\left(\frac{f(x)}{q}\right)=\sum_{x=1}^{q} \rho\left(\frac{f(x)}{q}\right) .
$$

Пользуясь формулой умножения можно упростить вывод следующей оценки для такой суммы.

ТЕОРема 4. При условии $\left(a_{n}, \ldots, a_{1}, q\right)=1$ верно неравенство

$$
\left|S\left(\frac{f(x)}{q}\right)\right| \ll q^{1-1 / n} .
$$

Также решен аналог задачи о сходимости интеграла в проблеме Терри.

ТеОРема 5. Пусть в предъдущих обозначениях

$$
A\left(p^{s}\right)=\sum_{\substack{0 \leqslant a_{i} \leqslant p^{s}-1 \\\left(a_{1}, \ldots, a_{n}, p\right)=1}}\left|p^{-s} S\left(\frac{f(x)}{p^{s}}\right)\right|^{2 k},
$$

u пусть $\sigma_{p}=1+\sum_{s \geqslant 1} A\left(p^{s}\right)$.

Тогда ряд, определяющий $\sigma_{p}$, сходится тогда и только тогда, когда

$$
2 k>\frac{n^{2}+n}{2}+1
$$

Кроме того, выполнен “дискретный” аналог теоремы 3. 
Теорема 6. Пусть $n, r \geqslant 2, p-$ простое число,

$$
F\left(x_{1}, \ldots, x_{r}\right)=\sum_{0 \leqslant t_{i} \leqslant n} a\left(t_{1}, \ldots, t_{r}\right) x_{1}^{t_{1}} \ldots x_{r}^{t_{r}} .
$$

$\operatorname{Ecли~}(a(0, \ldots, 1), \ldots, a(n, \ldots, n), p)=1, m o$

$$
\left|S\left(\frac{F\left(x_{1}, \ldots, x_{r}\right)}{p^{\alpha}}\right)\right| \ll_{n, r}(\alpha+1)^{r-1} p^{\alpha(r-1 / n)} .
$$

Доказан также вариант теоремы Виноградова о среднем.

TEOPEMA 7. Пусms

$$
J=\int_{[0,1]^{n+1}}\left|\sum_{x \leqslant P} \rho\left(\alpha_{0}+\alpha_{1} x+\cdots+\alpha_{n} x^{n}\right)\right|^{2 k} d \alpha_{0} d \alpha_{1} \ldots d \alpha_{n} .
$$

Если $\tau \geqslant 0$ целое, $k \geqslant n \tau, P \geqslant 1$ и $M \geqslant 1$, то

$$
J \leqslant D P^{2 k-n(n+1) / 2+\delta(\tau)},
$$

где

$$
\delta(\tau)=\frac{n(n+1)}{2}\left(1-\frac{1}{n}\right)^{\tau}, \quad D=D(\tau)=(n \tau)^{6 n \tau}(2 n)^{4 n(n+1) \tau} .
$$

И. Д. Шкредов (Математический институт им. В. А. Стеклова Российской академии наук, г. Москва), Суммы мультипликативных характеров от аддитивных сверток

Пусть $p$ - простое число и $\chi$ - неглавный мультипликативный характер по модулю $p$, $A, B \subset \mathbb{F}_{p}$ - подмножества. Зададимся вопросом: при каких условиях можно доказать нетривиальную оценку на величину

$$
\sigma(A, B)=\sum_{a \in A, b \in B} \chi(a+b)
$$

Иными словами, мы хотим доказывать, что при некоторых ограничениях на $A$ и $B$ выполнено $\sigma(A, B)=o(|A||B|)$. Первая оценка такого рода - это теорема Г. Дэвенпорта и П. Эрдеша, утверждающая, что справедлива оценка $\sigma(A, B)<\sqrt{|A||B| p}$, которая нетривиальна при $|A||B| \gg p$.

А. А. Карацуба доказал нетривиальную оценку в более слабых, но несимметричных ограничениях на $A$ и $B$, а именно

Теорема 1 (А. А. Карацуба, 1992). Пусть $\varepsilon_{1}, \varepsilon_{2}>0,|A|>p^{\varepsilon_{1}},|B|>p^{1 / 2+\varepsilon_{2}}$.

Тогда существует такая константа $\delta\left(\varepsilon_{1}, \varepsilon_{2}\right)>0$, ито

$$
\sigma(A, B) \ll|A||B| p^{-\delta\left(\varepsilon_{1}, \varepsilon_{2}\right)} .
$$

Карацуба также высказал гипотезу о том, что аналогичная оценка будет иметь место в случае $|A|=|B| \sim \sqrt{p}$. Имеется также более сильная гипотеза - гипотеза о графе Пэли, которая утверждает, что такая же оценка верна при $|A|,|B| \gg p^{\varepsilon}$. В случае $A=B$ и $\chi=\left(\frac{\dot{p}}{p}\right)$ она эквивалентна тому, что наибольшая клика в графе Пэли $\mathcal{G}=(\mathcal{V}, \mathcal{E})$, где $\mathcal{V}=\mathbb{F}_{p}$, а $x \sim y$ если $(x-y)$ - квадратичный вычет, не превосходит $p^{\varepsilon}$.

М.-Ч. Чанг доказала нетривиальные оценки на сумму $\sigma(A, B)$ для специальных множеств $A$ и $B$, например, следующую. 
Теорема 2 (М.-Ч. Чанг). Пусть $A$ - интервал, $|A|=p^{\alpha}$ и наименьшее расстояние между элементами $B$ оценивается снизу величиной $p^{\alpha}$, причем $|B|=p^{\beta}$. Тогда при выполнении условия $2 \alpha+\beta-\alpha \beta /(1-\alpha)>1 / 2+\delta$ имеет место оценка

$$
\left|\sum_{a \in A, b \in B} \chi(a+b)\right|<|A||B| p^{-\delta^{2} / 12} .
$$

Похожие оценки имеют место, если одно из множеств имеет малое удвоение.

Теорема 3 (М.-Ч. Чанг). Пусть $A, B \subset \mathbb{F}_{p}, \varepsilon>0, K \geqslant 1,|A|,|B|>p^{4 / 9+\varepsilon} u|A+A| \leqslant$ $K|A|$. Тогда выполнено неравенство

$$
\left|\sum_{a \in A, b \in B} \chi(a+b)\right| \ll_{K, \varepsilon}|A||B| p^{-\tau(K, \varepsilon)},
$$

где $\tau(K, \varepsilon)>0$.

Эта оценка нетривиальна при $K \ll \ln ^{c} p, c>0$.

Выполняется также следующая оценка Д. А. Бёрджесса для арифметических прогрессий длины более чем $p^{1 / 4+\varepsilon}$.

Теорема 4. Пусть $P \subseteq \mathbb{F}_{p}$ - арифметическая прогрессия, $|P|>p^{1 / 4+\varepsilon}$. Тогда для некоторого положительного $\delta(\varepsilon)>0$ выполнено неравенство

$$
\left|\sum_{x \in P} \chi(x)\right| \ll|P| p^{-\delta(\varepsilon)} .
$$

В 2015 г. Б. Хансон доказал оценку для тройной суммы в предположении, что мощности всех трех множеств больше $\delta \sqrt{p}$, где $\delta>0$.

Теорема 5. Если $|A|,|B|,|C|>\delta \sqrt{p}$, то справедливо равенство

$$
\left|\sum_{a \in A, b \in B, c \in C} \chi(a+b+c)\right|=o_{\delta}(|A||B||C|) .
$$

Зависимость от $\delta$ в этой теореме устроена крайне сложно. Однако докладчику удалось доказать теорему, улучшающую теоремы 3 и 5 , а именно

Теорема 6 (И.Д. Шкредов, 2016). Пусть $\varepsilon>0 u K \geqslant 1$. Если подмножества $A, B \subseteq \mathbb{F}_{p}$ таковы, что $|A|,|B|>p^{1 / 3+\varepsilon} u|A+A| \leqslant K|A|$, mо

$$
\left|\sum_{a \in A, b \in B} \chi(a+b)\right| \ll_{K, \varepsilon}|A||B| p^{-\tau(K, \varepsilon)},
$$

где $\tau(K, \varepsilon)>0$.

Доказательство в общих чертах следует логике доказательства Бёрджесса и опирается на следующий факт из аддитивной комбинаторики.

Теорема 7 (Э. Крут, О. Сисаск, 2010). Пусть $\varepsilon \in(0,1), K \geqslant 1, f: \mathbb{F}_{p} \rightarrow \mathbb{C} u|A+A| \leqslant$ $K|A|$.

Тогда существует подмножество $T \subseteq \mathbb{F}_{p}$ с условием $|T| \geqslant|A| e^{-O\left(\varepsilon^{-2} p \ln |K|\right)}$ и такое, что для всякого $t \in T$ выполнено следующее неравенство для $L^{p}$-норм:

$$
\left\|\left(f * 1_{A}\right)(x+t)-\left(f * 1_{A}\right)(x)\right\|_{p} \leqslant \varepsilon\|f\|_{p}|A|^{1 / p} .
$$


Как и в доказательстве Бёрджесса, естественно возникает вопрос о количестве решений некоторого уравнения, но в нашем случае это уравнение

$$
\left\{x\left(y_{1}+y_{2}\right)=z\left(w_{1}+w_{2}\right): x, z \in T, y_{i}, w_{i} \in A\right\} .
$$

Обозначим количество его решений через $N(T, A)$.

В 2015 г. рядом авторов была доказана верхняя оценка для $N(T, A)$.

Теорема 8 (Э. Аксой, Б. Мёрфи, М. Руднев, И. Д. Шкредов). Для любъх двух подмножеств $А$ и

$$
N(T, A) \ll|A|^{3}|T|^{3 / 2} .
$$

В доказательстве существенно используется сложная теорема М. Руднева об оценках для количества инцидентностей.

А. В. Шубин (Московский физико-технический институт (государственный университет), г. Долгопрудный Московской обл.), О дробных долях, связанных с функиией $N / x$

Классическая проблема делителей Дирихле теснейшим образом связана с уточнением асимптотической формулы

$$
\sum_{n \leqslant N}\left\{\frac{x}{n}\right\}=c N+O\left(x^{\alpha+\varepsilon}\right) .
$$

Если $N<x^{\beta}$ с $\beta<1$, то дробные доли равномерно распределены, и потому $c=1$, в случае же $N=x$ равнораспределенность исчезает, и получается $c=1-\gamma$.

Наилучшая на данный момент оценка остаточного члена доказана М. Хаксли: его результат гласит, что можно брать $\alpha=131 / 416$.

Докладчиком при помощи метода тригонометрических сумм были получены асимптотические формулы общего вида для сумм по $n \leqslant x$ (и более коротких сумм) $f(\{x / n\})$, где $f-$ любая достаточно хорошая функция, и таких же выражений с гладкой весовой функцией.

В случае короткой суммы имеет место равнораспределенность, поэтому верно такое утверждение.

ТЕОРЕма 1. Для $x>0$ и гладкой положительной монотонной функции $f$ верна асимптотическая формула

$$
\sum_{n \leqslant \sqrt{x}} f\left(\left\{\frac{x}{n}\right\}\right)=\sqrt{x} \int_{0}^{1} f(u) d u+O\left(x^{1 / 3} \ln ^{4 / 3} x\right) .
$$

Такая же формула верна и для сумм

$$
\sum_{n \leqslant \sqrt{x}} f\left(\left\{\frac{x}{n+v}\right\}\right), \quad v \in[0,1)
$$

В том же случае, когда $n$ пробегает отрезок $[1, x]$, интеграл по отрезку заменяется на несколько другой линейный функционал.

ТЕОРЕма 2. Для всякой положительной, монотонной и гладкой функции $f$ на отрезке выполнено равенство

$$
\sum_{n \leqslant x} f\left(\left\{\frac{x}{n}\right\}\right)=x c(f)+O\left(x^{1 / 3} \ln ^{4 / 3} x\right)
$$

где

$$
c(f)=\sum_{k \geqslant 1} \int_{0}^{1} \frac{f(u) d u}{(k+u)^{2}} .
$$


В случае сумм с весами получается такой факт.

Теорема 3. Пусть $f \in C^{1}[0,1]$ - монотонная положительная функиия, $\psi$ - гладкая, вещественная и положительная. Тогда

$$
S(f, \psi ; x)=\sum_{n \leqslant x} \psi(n) f\left(\left\{\frac{x}{n}\right\}\right)=x \sum_{k \geqslant 1} \int_{0}^{1} \frac{f(u) \psi(x /(k+u)) d u}{(k+u)^{2}}+R,
$$

гдe

$$
R \ll \inf _{2 \leqslant y \leqslant x} \max \left\{\sum_{k \leqslant y} \psi(k), \sum_{k \leqslant x / y} \psi\left(\frac{x}{k}\right), x \sum_{k>x / y} \frac{1}{k^{2}} \psi\left(\frac{x}{k}\right)\right\} .
$$

Применяя теоремы 1-3, можно вывести такие три асимптотические формулы.

Теорема 4. Пусть $g_{N}$ - количество целых $x, y, z, t$ с $x y=z t u x, y, z, t \leqslant N$. Тогда

$$
g_{N}=\frac{12}{\pi^{2}} N^{2} \ln N+N^{2}\left(\frac{12}{\pi^{2}}\left(2 \gamma-\frac{1}{2}-\frac{6}{\pi^{2}} \zeta^{\prime}(2)\right)-2\right)+O\left(N^{3 / 2}\right) .
$$

Если $\tau(k)$ - количество делителей числа $k$, то

$$
\sum_{k \leqslant x} \tau(k)\left\{\frac{x}{k}\right\}=(1-\gamma) x \ln x+B x+O\left(x^{2 / 3+\varepsilon}\right) .
$$

Наконеи, если $r_{2}(k)$ - количество способов представить $k$ в виде суммы двух квадратов иелых чисел, то

$$
\sum_{k \leqslant x} r_{2}(k)\left\{\frac{x}{k}\right\}=(1-\gamma) \pi x+O\left(x^{2 / 3}\right) .
$$

Также был получен ряд формул с суммами по специальным подмножествам в $\mathbb{N}$.

Теорема 5. Пусть $f \in C^{1}[0,1]$ - положительная монотонная функиия. Тогда для суммы по простым числам справедлива асимптотическая формула

$$
\sum_{p \leqslant x} f\left(\left\{\frac{x}{p}\right\}\right)=\int_{2}^{x} \frac{f(\{x / y\}) d y}{\ln y}+O_{f}\left(x e^{-c \sqrt{\ln x}}\right) .
$$

Аналогичная формула имеет место в случае суммирования по числам, представимым суммами двух квадратов. 